\title{
Vibration Analysis of a Cracked Rotor Surrounded by Viscous Liquid
}

\author{
R. K. BEHERA \\ D. R. K. PARHI \\ Department of Mechanical Engineering, National Institute of Technology, Rourkela, 769008, \\ Orissa,India(rabi57@rediffmail.com)

\section{S. K. SAHU} \\ Department of Civil Engineering, National Institute of Technology Rourkela, 769008, Orissa, \\ India
}

\begin{abstract}
Crack detection in, and vibration characteristics of, a shaft with two open cracks rotating in a fluid medium are studied in this paper. The influence coefficient method is used to find the fundamental frequency of the cracked shaft. The frequency contours with respect to crack depths and locations are used to identify cracks. The effect of fluid on the cracked rotor is analyzed with the help of Navier-Stokes equations. The dynamic response of the rotating cracked shaft is compared with that of an uncracked one, and it is found that, when the cracked rotor rotates in a viscous fluid, there is change in the critical speeds and amplitudes of vibration.
\end{abstract}

Keywords: crack depth, crack location, rotor, viscous fluid

\section{NOMENCLATURE}

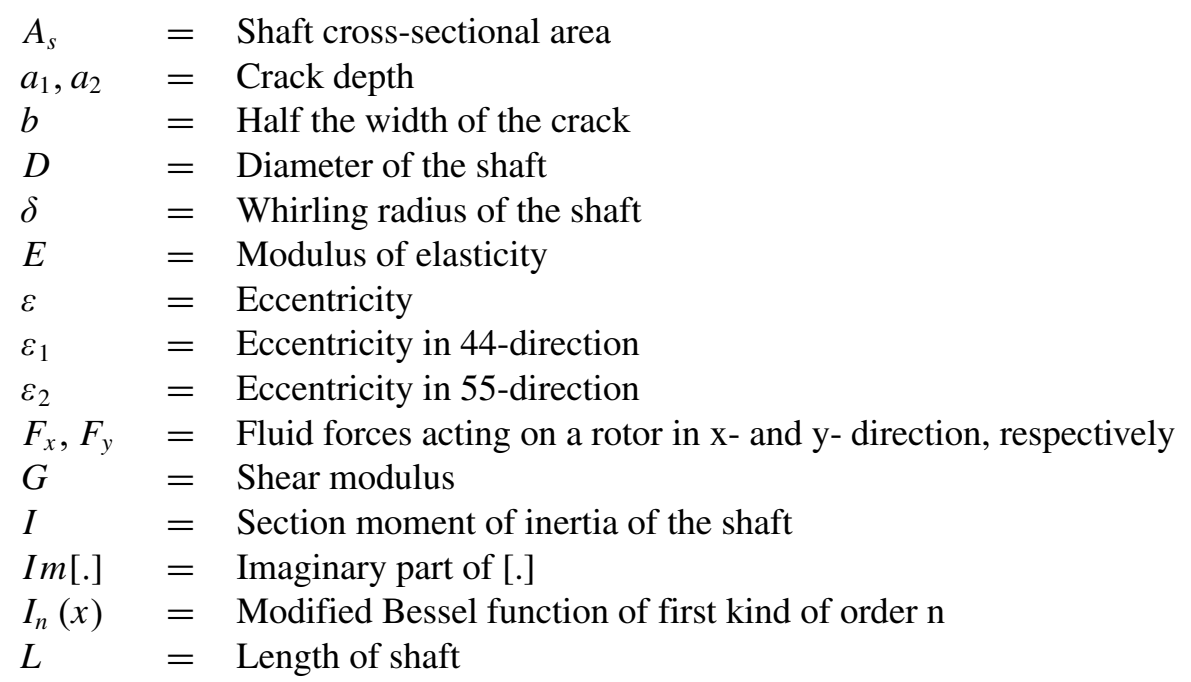




\begin{tabular}{|c|c|c|}
\hline$L_{1}, L_{2}$ & $=$ & Crack position from left end of the shaft \\
\hline$k$ & $=$ & $\sqrt{i \omega / \nu}$, where $i=\sqrt{-1.0}$ \\
\hline$K_{55}, K_{44}$ & $=$ & Stiffness of the cracked shaft in two directions (55- and 44-) \\
\hline$k k$ & $=$ & $6\left(1+v_{1}\right) /\left(7+6 v_{1}\right)$ \\
\hline$\left[K_{g}\right]$ & $=$ & Global stiffness matrix \\
\hline$K_{n}(x)$ & $=$ & Modified Bessel function of second kind of order $n$ \\
\hline$m_{s}$ & $=$ & Mass of the shaft per unit length \\
\hline$m$ & $=$ & Fluid mass displaced by the shaft per unit length \\
\hline$p$ & $=$ & pressure \\
\hline$R_{1}$ & $=$ & Radius of the shaft \\
\hline$R_{2}$ & $=$ & Radius of the container \\
\hline$t$ & $=$ & Time \\
\hline$u$ & $=$ & Radial flow velocity \\
\hline$v$ & $=$ & Tangential flow velocity \\
\hline$\alpha$ & $=$ & $k R_{1}$ \\
\hline$\beta$ & $=$ & $k R_{2}$ \\
\hline$\mu$ & $=$ & Coefficient of viscosity \\
\hline$v$ & $=$ & Coefficient of kinematic viscosity \\
\hline$v_{1}$ & $=$ & Poisson's ratio \\
\hline$\rho$ & $=$ & Fluid density \\
\hline$\rho_{s}$ & $=$ & Mass density of the shaft \\
\hline$\psi, \psi_{1}, \psi_{2}$ & $=$ & Stream functions \\
\hline$\omega$ & $=$ & Rotating speed \\
\hline$\omega_{o}$ & $=$ & Natural angular frequency of the uncracked rotor in air \\
\hline$\omega_{x x}, \omega_{y y}$ & $=$ & Critical speeds in the $x$ - and $y$ - directions, respectively \\
\hline$\Omega$ & $=$ & Angular velocity of whirling \\
\hline $\operatorname{Dir}^{\mathrm{n}}$ & $=$ & Direction \\
\hline 44-direction & $=$ & Direction perpendicular to crack depth \\
\hline 55-direction & $=$ & Direction parallel to crack depth \\
\hline
\end{tabular}

\section{INTRODUCTION}

Cracks in rotors may occur for a variety of reasons. They may be fatigue cracks that are created under service conditions as a result of the limited fatigue strength. They may be caused by mechanical damage. There may also be cracks inside the material, created during the manufacturing processes.

Cracks are a risk factor in the safe operation of a rotor, decreasing performance of machines in which they are present. Most breakdowns in modern machinery are due to fatigue of the material; for this reason, methods for the early detection and localization of cracks have been the subject of recent investigation. Wauer (1990) presented a review on the dynamics of rotor with cracks.

A crack in a rotor causes local variations in its stiffness. These changes affect the dynamics of the system: The frequencies of natural vibrations and amplitudes of forced vibration are changed by the existence of such cracks (Papadopoulos and Dimarogonas (1987)). In particular, the effects of a transverse crack which propagates from the surface of the shaft 
to its axis have been studied by Papadopoulos and Dimarogonas (1987), Wauer (1990), and Collins et al. (1991).The influence of transverse cracks on coupled torsional and bending vibrations of a rotor was studied by Ostachowicz and Krawczuk (1996).

When a cracked shaft rotates in a viscous medium, the analysis of critical speed becomes complex. Kito (1956) studied the effect of fluid forces on a eccentrically rotating circular rod in a liquid medium. In his analysis, he assumed the flow velocity distribution to be linear over a gap between the rod and cylinder. Iida (1958) examined the same problem, but using an infinitely extending water region. Fritz (1970) considered a circular rod rotating in a cylinder with a small gap between the rotor and cylinder, his analysis including the influence of turbulence and Taylor vortex flow. Brenner (1976) has analyzed theoretically the fluid forces acting on a circular rod rotating in a circular cylinder for high and low Reynolds numbers. Walston et al. (1964) established the dynamic behavior of an overhanging shaft submerged in a viscous fluid. The effects of the fluid upon the critical speed and amplitude of the rotor were determined experimentally, and a correlation equation for amplitude as a function of viscosity, velocity and mass developed with the help of statistical regression analysis. The analytical results on the fundamental critical speed of a shaft in a viscous fluid were studied by Shimogo and Kazao (1982).

Wauer et al. (1994) and Kadyrov et al. (2001) have studied the oscillations of a rigid cylinder in a cylindrical duct filled with a viscous incompressible fluid. They used mathematical analysis, presented the theoretical results for eigen frequency subjected to different fluid parameters.

In this paper, the dynamic response of a rotor with two cracks is investigated in a viscous medium. The results of the current analysis should be helpful in the dynamic study of (a) high-speed boring machines, (b) high-speed rotors in centrifuges, which are prone to fatigue, (c) turbine rotors, (d) rotors used for drilling oil from the sea bed, and (e) rotors of ships, as well as for preventing failure of rotors used in machines subjected to various environmental conditions.

\section{THEORETICAL ANALYSIS}

In Figure 1(b) a steady whirling motion of a rotating shaft is illustrated schematically. In Figure 1(a), point $\mathrm{O}^{\prime}$ designates an origin of a space-fixed co-ordinate system $\mathrm{x}-\mathrm{y}$, and the point $\mathrm{O}^{\prime}$ indicates the center of a rotating shaft. The whirling motion is represented by a rotating point $\mathrm{O}^{\prime}$ around the point $\mathrm{O}$ with a radius $\delta$ and a speed of rotation $\Omega$.

\subsection{Equation of Fluid Motion}

A shaft of cross sectional radius $R_{1}$, rotating with an angular speed $\omega$, having whirling speed $\Omega$ with $\delta$ as the whirling radius, is shown in Figures 1(a) and (b). The Navier-Stokes equation for fluid velocity in the polar co-ordinate system $r-\theta$ can be expressed as:

$$
\frac{\partial u}{\partial t}=-\frac{1}{\rho} \frac{\partial p}{\partial r}+v\left(\frac{\partial^{2} u}{\partial r^{2}}+\frac{1}{r} \frac{\partial u}{\partial r}-\frac{u}{r^{2}}+\frac{1}{r^{2}} \frac{\partial^{2} u}{\partial \theta^{2}}-\frac{2}{r^{2}} \frac{\partial v}{\partial \theta}\right)
$$




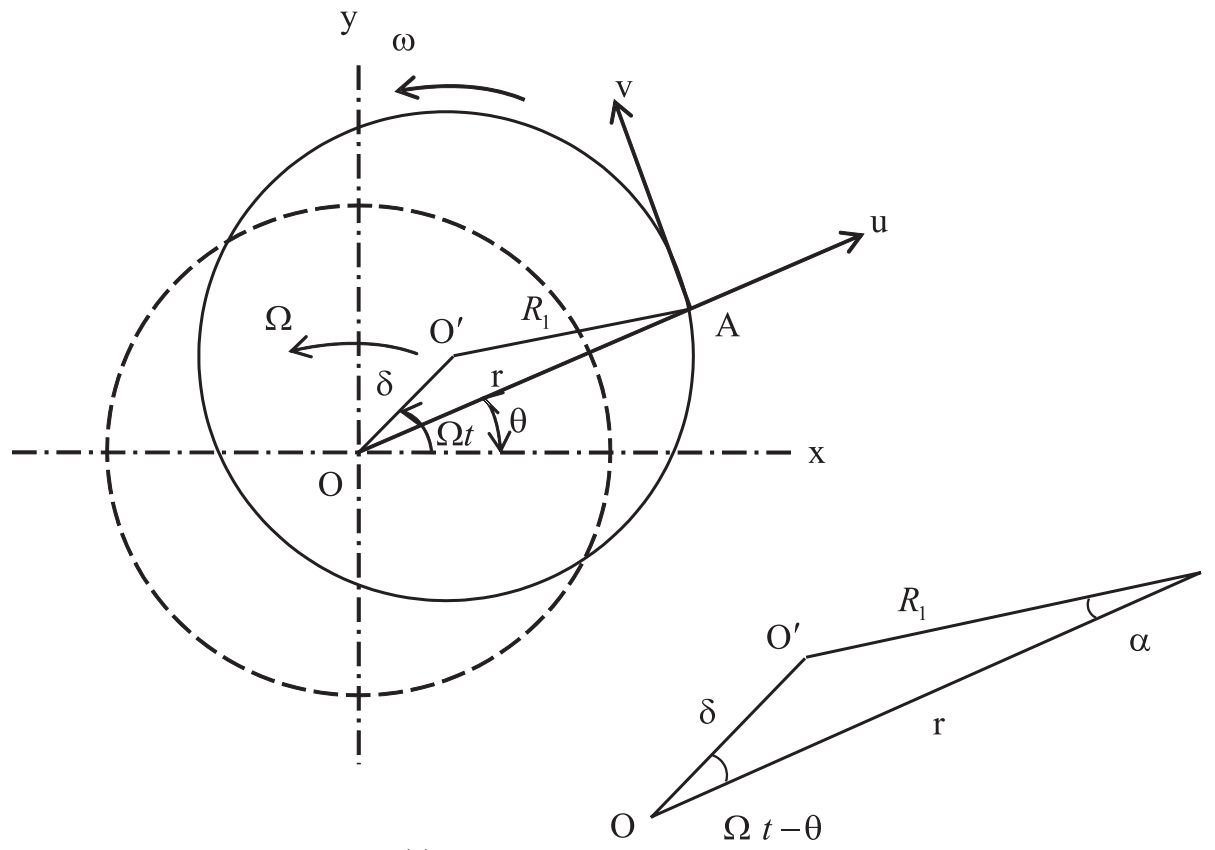

(a)

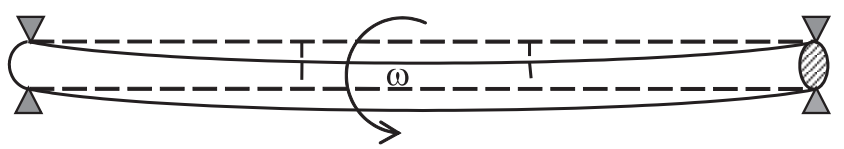

(b)

Figure 1. (a) Two dimensional model of a whirling rotor, (b) A whirling rotor with two cracks.

$$
\frac{\partial v}{\partial t}=-\frac{1}{\rho r} \frac{\partial p}{\partial \theta}+v\left(\frac{\partial^{2} u}{\partial r^{2}}+\frac{1}{r} \frac{\partial v}{\partial r}-\frac{v}{r^{2}}+\frac{1}{r^{2}} \frac{\partial^{2} v}{\partial \theta^{2}}+\frac{2}{r^{2}} \frac{\partial v}{\partial \theta}\right)
$$

Where $\mathrm{u}$ and $\mathrm{v}$ denote flow velocities in the radial and tangential directions respectively, and $p$ is the fluid pressure. With the help of the stream function $\psi(r, \theta, t)$ and eliminating the pressure terms, the above equations can be written as

$$
\nabla^{4} \psi-\frac{1}{v} \frac{\partial}{\partial t}\left(\nabla^{2} \psi\right)=0
$$

where $\nabla^{2}=\frac{\partial^{2}}{\partial r^{2}}+\frac{1}{r} \frac{\partial}{\partial r}+\frac{1}{r^{2}} \frac{\partial^{2}}{\partial \theta^{2}}$ 
Equation (2) can be divided into two parts

$$
\nabla^{2} \psi=0, \quad \nabla^{2} \psi-\left(\frac{1}{v}\right)\left(\frac{\partial \psi}{\partial t}\right)=0
$$

The solution to equation (2) is given by

$$
\psi=\psi_{1}+\psi_{2}
$$

Where $\psi_{1}$ and $\psi_{2}$ are solutions of equation (3).

The radial and tangential components of the flow velocity at point $A$ on the surface of shaft in Figure 1 are

$$
\begin{aligned}
& u_{A}=R_{1} \omega \sin \alpha-\delta \Omega \sin (\Omega t-\theta) \\
& v_{A}=R_{1} \omega \cos \alpha-\delta \Omega \cos (\Omega t-\theta)
\end{aligned}
$$

where $\alpha$ is the angle between the lines $\mathrm{O}^{\prime} \mathrm{A}$ and $\mathrm{OA}$. Considering the relationships

$$
\sin \alpha=\left(\delta / R_{1}\right) \sin (\Omega t-\theta) \text { and } \cos \alpha=1 \text { for } \delta \ll R_{1}
$$

for $r=R_{1}$ the equation (4) can be rewritten as

$$
\begin{aligned}
& \left.u\right|_{r=R_{1}}=\delta(\omega-\Omega) \sin (\Omega t-\theta)=\operatorname{Re}\left[-i \delta(\omega-\Omega) e^{i(\Omega t-\theta)}\right] \\
& \left.v\right|_{r=R_{1}}=\delta \Omega \cos (\Omega t-\theta)+R_{1} \omega=\operatorname{Re}\left[\delta \Omega e^{i(\Omega t-\theta)}\right]+R_{1} \omega
\end{aligned}
$$

where $i=\sqrt{-1}$ and $\operatorname{Re}$ [.] denotes the real part of [.]. For the special case $\omega=\Omega$ (i.e., for synchronous whirl) equation (5) reduces to

$$
\left.u\right|_{r=R_{1}}=0,\left.\quad v\right|_{r=R_{1}}=\operatorname{Re}\left[\delta \omega e^{i(\omega t-\theta)}\right]+R_{1} \omega
$$

When the shaft is immersed in an infinitely extending fluid region, the boundary condition for $r \rightarrow \infty\left(r=R_{2} \rightarrow \infty\right.$, i.e., the container radius is taken as $\left.R_{2} \rightarrow \infty\right)$ is

$$
\left.u\right|_{r=R_{2}=\infty}=\left.v\right|_{r=R_{2}=\infty}=0
$$

For the above conditions, the nonstationary components of the solutions $\psi_{1}$ and $\psi_{2}$ can be expressed as

$$
\psi_{1}(r, \theta, t)=F_{1}(r) e^{i(\omega t-\theta)}, \quad \psi_{2}(r, \theta, t)=F_{2}(r) e^{i(\omega t-\theta)}
$$

From equations (8) and (3), one can obtain

$$
\frac{\mathrm{d}^{2} F_{1}(r)}{\mathrm{d} r^{2}}+\frac{1}{r} \frac{\mathrm{d} F_{1}(r)}{\mathrm{d} r}-\frac{1}{r^{2}} F_{1}(r)=0
$$




$$
\frac{\mathrm{d}^{2} F_{2}(r)}{\mathrm{d} r^{2}}+\frac{1}{r} \frac{\mathrm{d} F_{2}(r)}{\mathrm{d} r}-\left(\frac{1}{r^{2}}+k^{2}\right) F_{2}(r)=0, \quad\left(k=\sqrt{\frac{i \omega}{v}}\right)
$$

Since equation (9a) is Euler's equation and equation (9b) is Bessel's equation, the general solutions of these equations can be written as

$$
F_{1}(r)=\delta \omega\left(\frac{A_{q} R_{1}^{2}}{r}+B_{q} r\right), \quad F_{2}(r)=\delta \omega R_{1}\left\{C_{q} I_{1}(k r)+D_{q} K_{1}(k r)\right\}
$$

where $A_{q}, B_{q}, C_{q} \& D_{q}$ are arbitrary constants, and $I_{1}(k r)$ and $K_{1}(k r)$ are modified Bessel functions of the first and second kinds respectively. Thus the nonstationary components of flow velocities induced by the whirling motion of a shaft are given as follows:

$$
\begin{aligned}
u_{r} & =i \delta \omega\left[A_{q}\left(\frac{R_{1}}{r}\right)^{2}+B_{q}+C_{q} \frac{R_{1}}{r} I_{1}(k r)+D_{q} \frac{R_{1}}{r} K_{1}(k r)\right] e^{i(\omega t-\theta)} \\
v_{r} & =\delta \omega\left[-A_{q}\left(\frac{R_{1}}{r}\right)^{2}+B_{q}+C_{q}\left\{-\left(\frac{R_{1}}{r}\right) I_{1}(k r)+k R_{1} I_{0}(k r)\right\}\right. \\
& \left.+D_{q}\left\{-\left(\frac{R_{1}}{r}\right) K_{1}(k r)-k R_{1} K_{0}(k r)\right\}\right] e^{i(\omega t-\theta)}
\end{aligned}
$$

\subsection{Fluid Forces}

Substituting the flow velocities given by equation (11) into equation (1), the nonstationary component of the pressure $p$ can be written as

$$
p=\int \frac{\partial p}{\partial \theta} \mathrm{d} \theta=\delta \rho \omega^{2}\left\{-\frac{A_{q} R_{1}^{2}}{r}+B_{q} r\right\} e^{i(\omega t-\theta)}
$$

Normal stress $\tau_{r r}$ and tangential stress $\tau_{r \theta}$ produced by the flow are consequently obtained as

$$
\begin{aligned}
\tau_{r r} & =-p+2 \mu \frac{\partial u_{r}}{\partial r}=i \mu \delta \omega\left[-\frac{4 A_{q} R_{1}^{2}}{r^{2}}+C_{q}\left\{-\frac{4 R_{1}}{r^{2}} I_{1}(k r)+\frac{2 k R_{1}}{r} I_{0}(k r)\right\}\right. \\
& \left.+D_{q}\left(-\frac{4 R_{1}}{r^{2}} K_{1}(k r)-\frac{2 k R_{1}}{r} K_{0}(k r)\right)\right] e^{i(\omega t-\theta)}+\delta \rho \omega^{2}\left\{\frac{A_{q} R_{1}^{2}}{r}-B_{q} r\right\} e^{i(\omega t-\theta)}(13 a \\
\tau_{r \theta} & =\mu\left\{r \frac{\partial}{\partial r}\left(\frac{v_{r}}{r}\right)+\frac{1}{r} \frac{\partial v_{r}}{\partial \theta}\right\} \\
& =\mu \delta \omega\left[\frac{4 A_{q} R_{1}}{r^{3}}+C\left\{\left(\frac{4 R_{1}}{r^{2}}+k^{2} R_{1}\right) I_{1}(k r)-\frac{2 k R_{1}}{r} I_{0}(k r)\right\}\right. \\
& \left.+D_{q}\left\{\left(\frac{4 R_{1}}{r^{2}}+k^{2} R_{1}\right) K_{1}(k r)+\frac{2 k R_{1}}{r} K_{0}(k r)\right\}\right] e^{i(\omega t-\theta)}
\end{aligned}
$$


Fluid forces acting on the surface (i.e., $r=R_{1}$ ) per unit length of the shaft in $\mathrm{x}$ - and $\mathrm{y}$ directions are obtained by integrating equation (13), giving

$$
\begin{aligned}
F_{x} & =\int_{0}^{2 \pi}\left(\tau_{r r} \cos \theta-\tau_{r \theta} \sin \theta\right) R_{1} \mathrm{~d} \theta \\
& =m \delta \omega^{2}\left[A_{q}-B_{q}-C_{q} I_{1}\left(\alpha_{1}\right)-D_{q} K_{1}\left(\alpha_{1}\right)\right] e^{i \omega t} \\
F_{y} & =\int_{0}^{2 \pi}\left(\tau_{r r} \sin \theta+\tau_{r \theta} \cos \theta\right) R_{1} \mathrm{~d} \theta \\
& =-i m \delta \omega^{2}\left[A_{q}-B_{q}-C_{q} I_{1}\left(\alpha_{1}\right)-D_{q} K_{1}\left(\alpha_{1}\right)\right] e^{i \omega t}
\end{aligned}
$$

Where $\alpha_{1}=k R_{1}$, and $m=\rho \pi R_{1}^{2}$ is the fluid mass displaced by the shaft per unit length. Since only the real parts of equation (14) are physically meaningful, the fluid forces $F_{x}$ and $F_{y}$ can be rewritten to give

$$
\begin{aligned}
& F_{x}=m \delta \omega^{2}[\operatorname{Re}(H) \cos \omega t-\operatorname{Im}(H) \sin \omega t] \\
& F_{y}=m \delta \omega^{2}[\operatorname{Re}(H) \sin \omega t+\operatorname{Im}(H) \cos \omega t]
\end{aligned}
$$

Where $H=A_{q}-B_{q}-C_{q} I_{1}\left(\alpha_{1}\right)-D_{q} K_{1}\left(\alpha_{1}\right)$ and $\operatorname{Re}(\mathrm{H})$ and $\operatorname{Im}(\mathrm{H})$ represent the real and imaginary part of parameter $\mathrm{H}$.

As the $\mathrm{x}$ - and $\mathrm{y}$ - coordinates of the shaft center are $x=\delta \cos \omega t$ and $y=\delta \sin \omega t$, equation (15) can be further rewritten into the following forms

$$
\begin{aligned}
& F_{x}=-m \operatorname{Re}(H) \frac{\mathrm{d}^{2} x}{\mathrm{~d} t^{2}}+m \omega \operatorname{Im}(H) \frac{\mathrm{d} x}{\mathrm{~d} t} \\
& F_{y}=-m \operatorname{Re}(H) \frac{\mathrm{d}^{2} y}{\mathrm{~d} t^{2}}+m \omega \operatorname{Im}(H) \frac{\mathrm{d} y}{\mathrm{~d} t}
\end{aligned}
$$

In equation (16), $m \operatorname{Re}(H)$ means virtual mass or added mass of fluid relating to the inertial force of the shaft, and $-m \omega \operatorname{Im}(H)$ denotes the viscous damping coefficient.

\subsection{Analysis of Rotor Motion}

Here, a simply supported rotating cracked shaft immersed in the fluid region is considered. The equations of motion for the shaft (with uniformly distributed mass and stiffness) are

$$
\begin{aligned}
& m_{s} \frac{\partial^{2}(x+\varepsilon \cos \omega t)}{\partial t^{2}}+E I \frac{\partial^{4} x}{\partial z^{4}}=F_{x} \\
& m_{s} \frac{\partial^{2}(y+\varepsilon \sin \omega t)}{\partial t^{2}}+E I \frac{\partial^{4} y}{\partial z^{4}}=F_{y}
\end{aligned}
$$


Where $m_{s}$ is the shaft mass per unit length, $E I$ is the bending stiffness of the shaft and $\varepsilon$ denotes the distance between the shaft center and the Center of Inertia of the rotor. Internal damping of the shaft is neglected.

Substituting equation (16) into equation (17), the equation of motion for the rotor in fluid can be obtained as

$$
\begin{aligned}
& \left(m_{s}+m \operatorname{Re}(H)\right) \frac{\partial^{2} x}{\partial t^{2}}-m \omega \operatorname{Im}(H) \frac{\partial x}{\partial t}+E I \frac{\partial^{4} x}{\partial z^{4}}=m_{s} \varepsilon \omega^{2} \cos \omega t \\
& \left(m_{s}+m \operatorname{Re}(H)\right) \frac{\partial^{2} y}{\partial t^{2}}-m \omega \operatorname{Im}(H) \frac{\partial y}{\partial t}+E I \frac{\partial^{4} y}{\partial z^{4}}=m_{s} \varepsilon \omega^{2} \sin \omega t
\end{aligned}
$$

The analysis is carried out using eccentricities $\varepsilon_{1}$ (along 44-direction) and $\varepsilon_{2}$ (along 55direction) as shown in Figure 2.

Introducing dimensionless quantities $\xi=x / R_{1}, \eta=y / R_{1}, \zeta=z / R_{1}, \varepsilon_{1}^{*}=\varepsilon_{1} / R_{1}$, $L^{*}=L / R_{1}, m^{*}=m / m_{s}, \omega_{1}^{*}=\omega / \omega_{x x}, \omega_{2}^{*}=\omega / \omega_{y y}, f_{1}=\left(\omega_{0} / \omega_{x x}\right)^{2}, f_{2}=\left(\omega_{0} / \omega_{y y}\right)^{2}$, $\tau_{1}=\omega_{x x} t, \tau_{2}=\omega_{y y} t$ (where $\omega_{x x}, \omega_{y y}$ are the fundamental natural frequencies of the cracked shaft in the $\mathrm{x}$ and $\mathrm{y}$ directions, respectively, (see Appendix A) as shown in Figure 2, and $\omega_{0}$ is the fundamental natural frequencie of the uncracked shaft $=\pi^{2}\left(E I / m_{s} L^{4}\right)^{0.5}$ ), equation (18) can be written as

$$
\begin{aligned}
& \left\{1+m^{*} \operatorname{Re}(H)\right\} \frac{\partial^{2} \xi}{\partial \tau_{1}^{2}}-m^{*} \omega_{1}^{*} \operatorname{Im}(H) \frac{\partial \xi}{\partial \tau_{1}}+f_{1} \frac{L^{* 4}}{\pi^{4}} \frac{\partial^{4} \xi}{\partial \zeta^{4}}=\varepsilon_{1}^{*}\left(\omega_{1}^{*}\right)^{2} \cos \left(\omega_{1}^{*} \tau_{1}\right) \\
& \left\{1+m^{*} \operatorname{Re}(H)\right\} \frac{\partial^{2} \eta}{\partial \tau_{2}^{2}}-m^{*} \omega_{2}^{*} \operatorname{Im}(H) \frac{\partial \eta}{\partial \tau_{2}}+f_{2} \frac{L^{* 4}}{\pi^{4}} \frac{\partial^{4} \eta}{\partial \zeta^{4}}=\varepsilon_{1}^{*}\left(\omega_{2}^{*}\right)^{2} \sin \left(\omega_{2}^{*} \tau_{2}\right)
\end{aligned}
$$

Applying the Fourier Transform to both sides of equation (19), we obtain

$$
\begin{aligned}
\ddot{X}_{v}+C_{01} \dot{X}_{v}+K_{v 1} X_{v} & =A_{v 1} \cos \left(\omega_{1}^{*} \tau_{1}\right) \\
A_{v 1} & =\frac{(2 / \nu \pi) \varepsilon_{1}^{*}\left(\omega_{1}^{*}\right)^{2} L^{*} \sin ^{2}(\nu \pi / 2)}{1+m_{1}^{*} \operatorname{Re}(H)} \\
\ddot{Y}_{v}+C_{02} \dot{Y}_{v}+K_{v 2} Y_{v} & =A_{\nu 2} \sin \left(\omega_{2}^{*} \tau_{2}\right), \\
A_{v 2} & =\frac{(2 / \nu \pi) \varepsilon_{1}^{*}\left(\omega_{2}^{*}\right)^{2} L^{*} \cos ^{2}(\nu \pi / 2)}{1+m_{2}^{*} \operatorname{Re}(H)}
\end{aligned}
$$

where $X_{v}\left(\tau_{1}\right)=\int_{0}^{L^{*}} \xi\left(\zeta, \tau_{1}\right) \sin \left(\nu \pi \zeta / L^{*}\right) \mathrm{d} \zeta, Y_{\nu}\left(\tau_{2}\right)=\int_{0}^{L^{*}} \eta\left(\zeta, \tau_{2}\right) \sin \left(\nu \pi \zeta / L^{*}\right) \mathrm{d} \zeta$, $C_{0 n}=\frac{-m_{n}^{*} \omega_{n}^{*} \operatorname{Im}(H)}{1+m_{n}^{*} \operatorname{Re}(H)}, K_{v n}=\frac{f_{n}}{1+m_{n}^{*} \operatorname{Re}(H)}$, [for $n=1,2$ and $\left.v=1,2, \ldots\right], f_{n}=f_{1}$ or $f_{2}$ for $n=1$ or 2

The steady state solution of equation (20) is easily obtained as $X_{v}\left(\tau_{1}\right)=(1 / 2) \delta_{v}^{*}$ $\cos \left(\omega_{1}^{*} \tau_{1}-\phi_{1}\right), Y_{v}\left(\tau_{2}\right)=(1 / 2) \delta_{v}^{*} \cos \left(\omega_{2}^{*} \tau_{2}-\phi_{2}\right)$ (for $\left.v=1,2 \ldots\right)$ where 


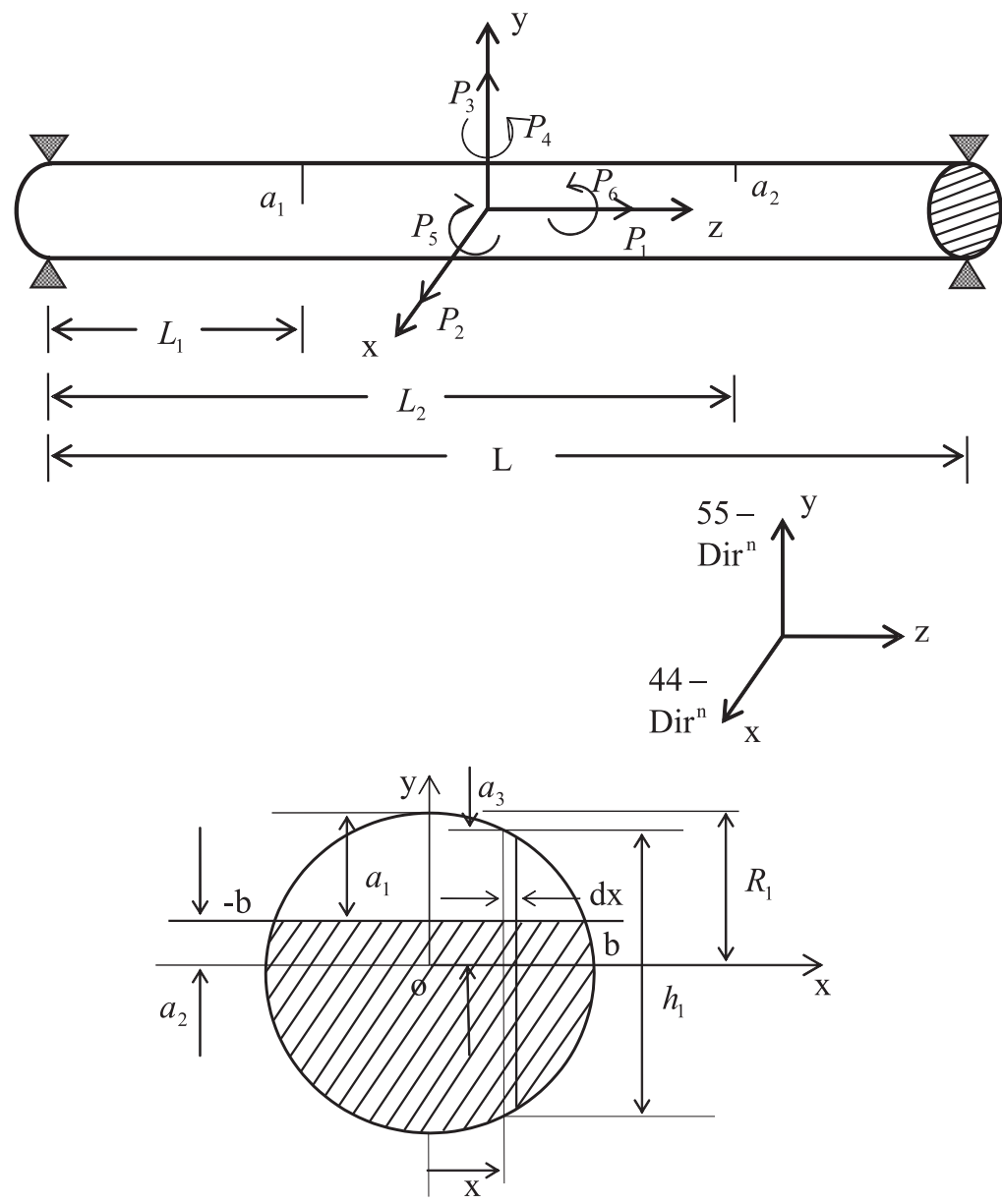

Cross Sectional View of the shaft at the position of crack

Figure 2. Simply supported shaft with cracks.

$$
\delta_{v n}^{*}=\frac{A_{v n}}{\sqrt{\left(K_{v n}-\left(\omega_{n}^{*}\right)^{2}\right)^{2}+\left(C_{0 n} \omega_{n}^{*}\right)^{2}}}, \quad \phi_{v n}=\tan ^{-1}\left(\frac{C_{0 n} \omega_{n}^{*}}{K_{v n}-\left(\omega_{n}^{*}\right)^{2}}\right)
$$

for $v=1,2, \ldots$, and $n=1$ or 2 .

Taking the inverse Fourier Transform of $X_{v}$, we get

$$
\xi\left(\zeta, \tau_{1}\right)=2 \sum_{v=1}^{\infty} X_{v}\left(\tau_{1}\right) \sin \left(v \pi \zeta / L^{*}\right)
$$

(and similarly for $Y_{v}$ ). 
From the above equations, the whirling motion for fundamental bending mode in $\mathrm{x}$ and y directions can be written, respectively, as

$$
\begin{aligned}
& \xi\left(\frac{L^{*}}{2}, \tau_{1}\right)=\delta_{11}^{*} \cos \left(\omega_{1}^{*} \tau_{1}-\phi_{11}\right) \\
& \eta\left(\frac{L^{*}}{2}, \tau_{2}\right)=\delta_{12}^{*} \sin \left(\omega_{2}^{*} \tau_{2}-\phi_{12}\right)
\end{aligned}
$$

where $\xi$ and $\eta$ are the dimensionless deflections in $\mathrm{x}$ and $\mathrm{y}$ directions, respectively, due to eccentricity $\varepsilon_{1}$ (eccentricity in the 44-direction, i.e., perpendicular to the crack).

When the 44-direction axis coincides with the $\mathrm{x}$ axis, the amplitude contribution of $\varepsilon_{1}$ is

$$
\begin{aligned}
& \xi_{44}\left(\frac{L^{*}}{2}, \tau_{1}\right)=\delta_{11}^{*} \cos \left(-\phi_{11}\right) \quad \text { in the } x \text { direction } \\
& \eta_{44}\left(\frac{L^{*}}{2}, \tau_{2}\right)=\delta_{12}^{*} \sin \left(\omega_{2}^{*} \tau_{2}-\phi_{12}\right), \text { when } \omega_{1}^{*} \tau=0 \quad \text { in the } y \text { direction }
\end{aligned}
$$

Similarly, the expression for $\xi\left(\frac{L^{*}}{2}, \tau_{1}\right)$ and $\eta\left(\frac{L^{*}}{2}, \tau_{1}\right)$ due to eccentricity $\varepsilon_{2}$ (eccentricity in the direction of the crack, i.e., the 55-direction) can be found by adopting the above procedure.

When the 55-direction axis coincides with the y axis, the amplitude contribution of $\varepsilon_{1}$ in the $\mathrm{x}$ and y directions is $\xi_{55}\left(\frac{L^{*}}{2}, \tau_{1}\right)$ and $\eta_{55}\left(\frac{L^{*}}{2}, \tau_{2}\right)$, respectively.

The total dimensionless deflection in $\mathrm{x}$ and $\mathrm{y}$ directions, when the 44-direction (perpendicular to the crack) and 55-direction (along the crack) coincide respectively with the $\mathrm{x}$ axis and $y$ axis are

$$
\begin{array}{lll}
\delta_{1}^{*}=\delta_{44}^{*}=\xi_{44}\left(\frac{L^{*}}{2}, \tau_{1}\right)+\xi_{55}\left(\frac{L^{*}}{2}, \tau_{1}\right) & \text { Along the } \mathrm{x}(44-) \text { direction } \\
\delta_{2}^{*}=\delta_{55}^{*}=\eta_{44}\left(\frac{L^{*}}{2}, \tau_{1}\right)+\eta_{55}\left(\frac{L^{*}}{2}, \tau_{1}\right) & \text { Along the y (55-) direction }
\end{array}
$$

$\delta_{1}^{*}\left(=\delta_{44}^{*}\right)$ and $\delta_{2}^{*}\left(=\delta_{55}^{*}\right)$ are the dimensionless amplitudes of the cracked rotor when the 44-direction and 55-direction coincide with the $\mathrm{x}$ axis and $\mathrm{y}$ axis, respectively.

\section{NUMERICAL RESULTS}

A simply supported cracked mild steel shaft with uniform cross-section area, length $=1.5$ $\mathrm{m}$, radius $=0.01 \mathrm{~m}$, modulus of elasticity $E=200 \mathrm{Gpa}$, poisons ratio $v_{1}=0.3$, and density $\rho=7860 \mathrm{~kg} / \mathrm{m}^{3}$ is considered for numerical analysis. Figure 2 shows the cracked shaft with various coupling forces. The different crack locations (from the left end of the shaft) are used to evaluate effects of crack location on modal properties of the shaft. The crack depths are chosen such that $a_{1,2} / D=0.1,0.2$, and 0.35 . The variation of the eigen values of 


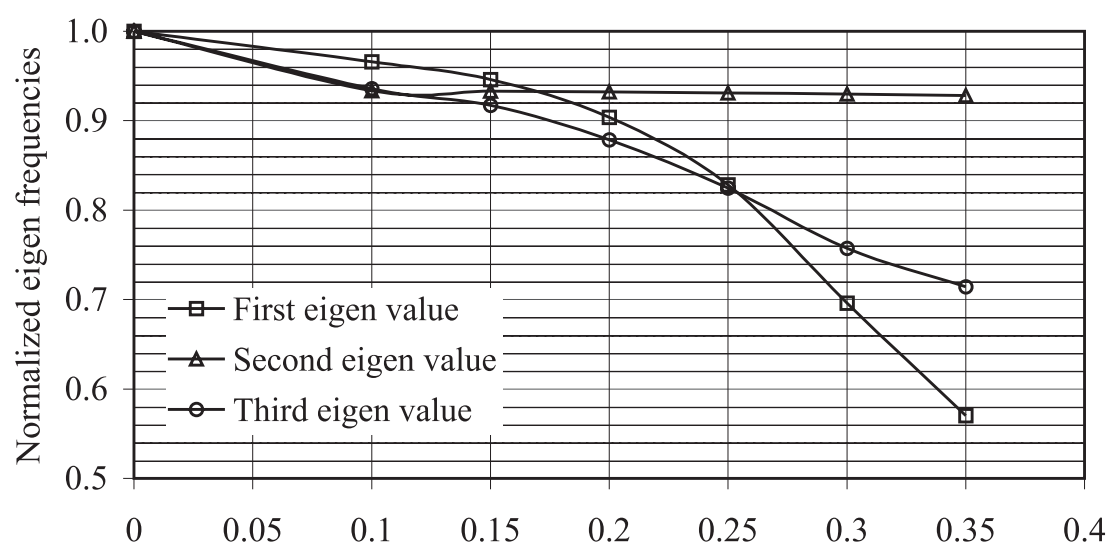

Relative crack depth

Figure 3. Variation of eigen values of cracked shaft against relative crack depth $\left(a_{2} / D\right) . R_{1} / L=0.0667$, $a_{1} / D=0.2, L_{1} / L=0.2, L_{2} / L=0.5$.

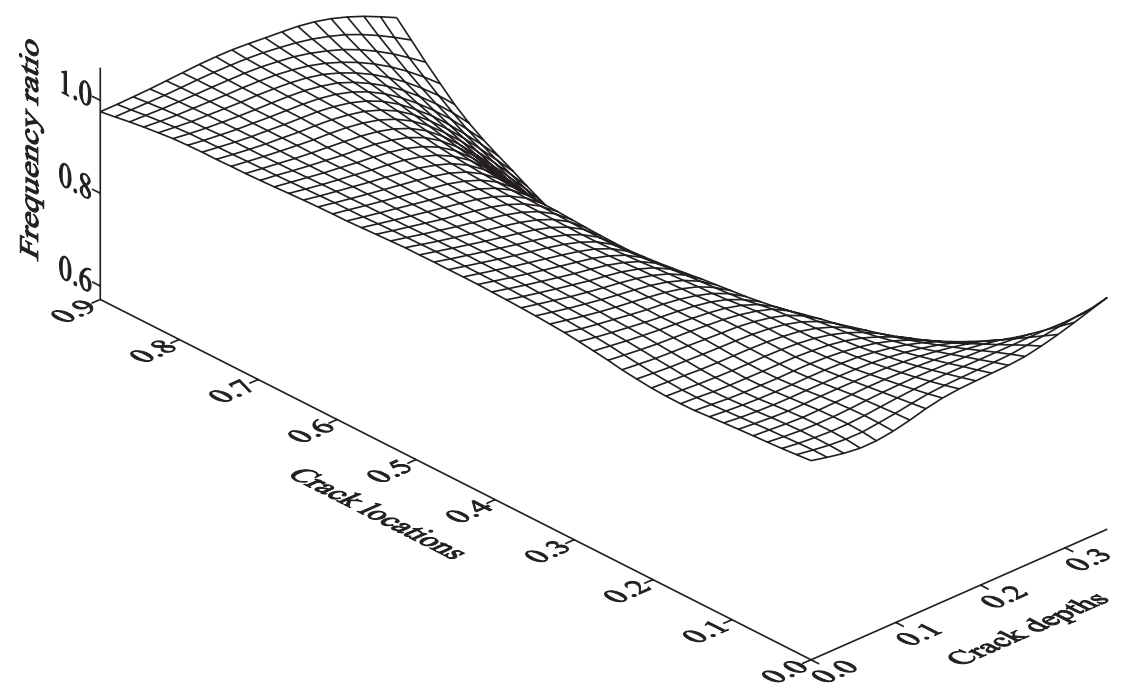

Figure 4. Frequencies against crack locations $\left(L_{2} / L\right)$ and depths $\left(a_{2} / D\right)$ for first mode of vibration. $a_{1} / D=0.1, L_{1} / L=0.1667$.

the cracked shaft for different relative crack depths $\left(a_{2} / D\right)$ is shown in Figure 3. In Figure 3 the relative crack depth $\left(a_{1} / D\right)$ at the first location $\left(L_{1} / L\right)$ is arbitrarily taken as 0.1 . The slenderness ratio $\left(R_{1} / L\right)$ is taken as 0.0667 . It is observed that the natural frequencies of the rotor decrease with increasing relative crack depths.

Figures 4 to 6 show the normalized frequencies against crack location and depth in 3-dimensional plots for the first, second and third natural frequencies (respectively) corresponding to various crack conditions. From these figures, it can be seen that the crack 


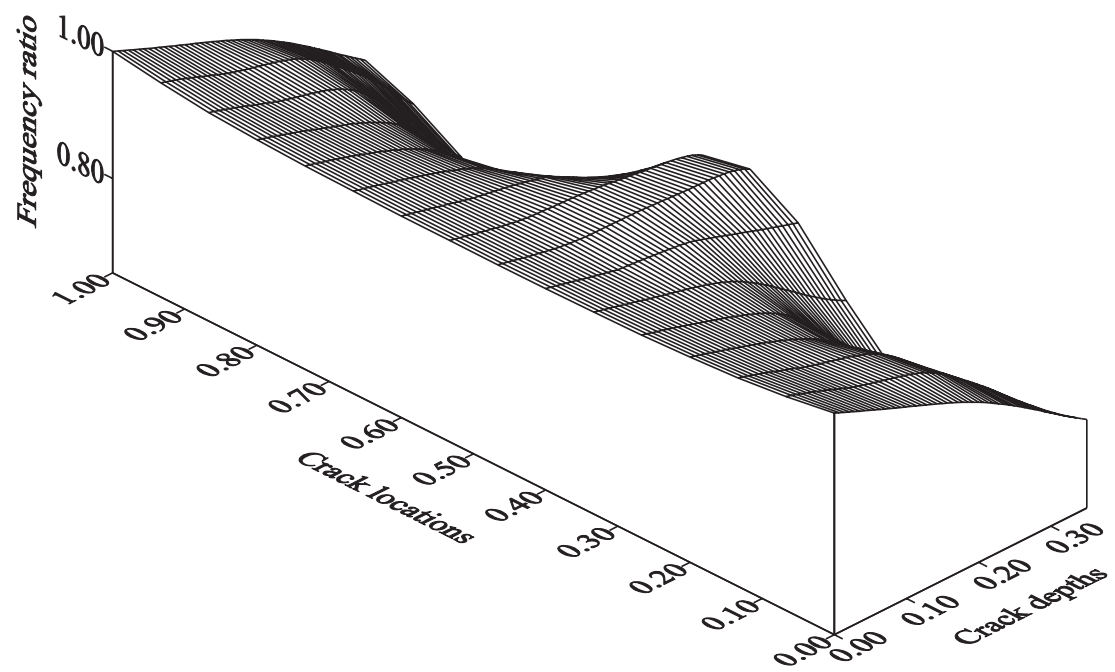

Figure 5. Frequencies against crack locations $\left(L_{2} / L\right)$ and depths $\left(a_{2} / D\right)$ for second mode of vibration. $a_{1} / D=0.1, L_{1} / L=0.1667$.

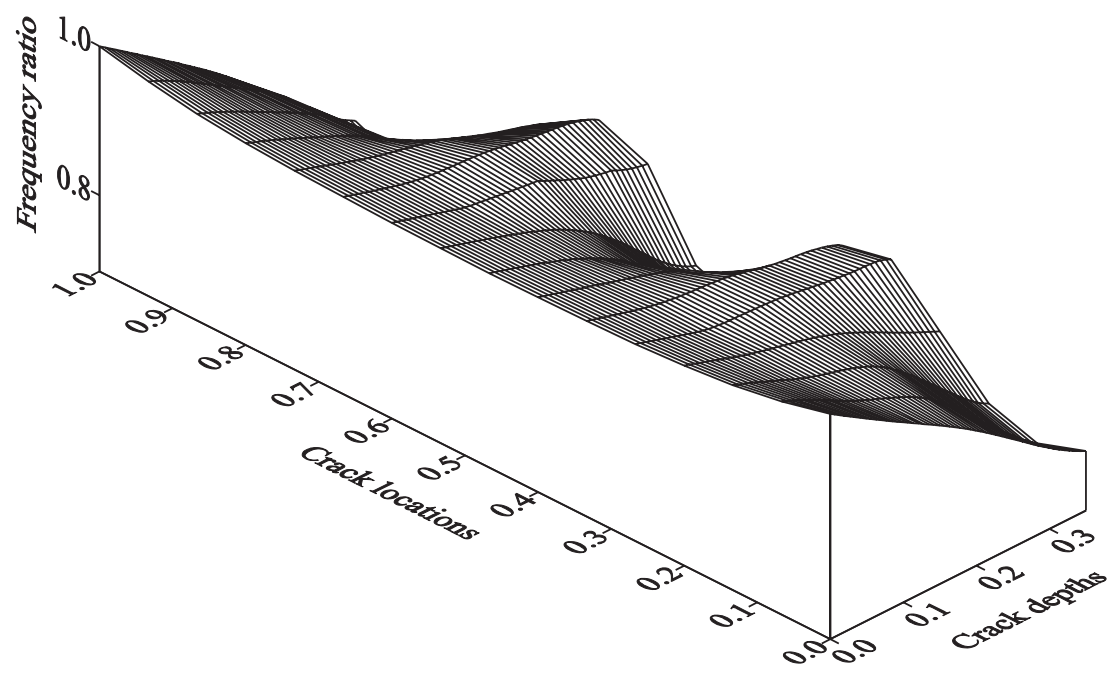

Figure 6. Frequencies against crack locations $\left(L_{2} / L\right)$ and depths $\left(a_{2} / D\right)$ for third mode of vibration. $a_{1} / D=0.1, L_{1} / L=0.1667$.

location and crack depth ratios are directly related to the frequency ratios. It will also be noticed that presence of cracks near the ends of the shaft does not change the frequencies. For the first mode (Figure 4) the maximum change of frequency takes place when the crack occurs at the center. From Figures 5 and 6 it can be seen that a crack located on a nodal point has a minimal effect on the frequencies of that mode. 


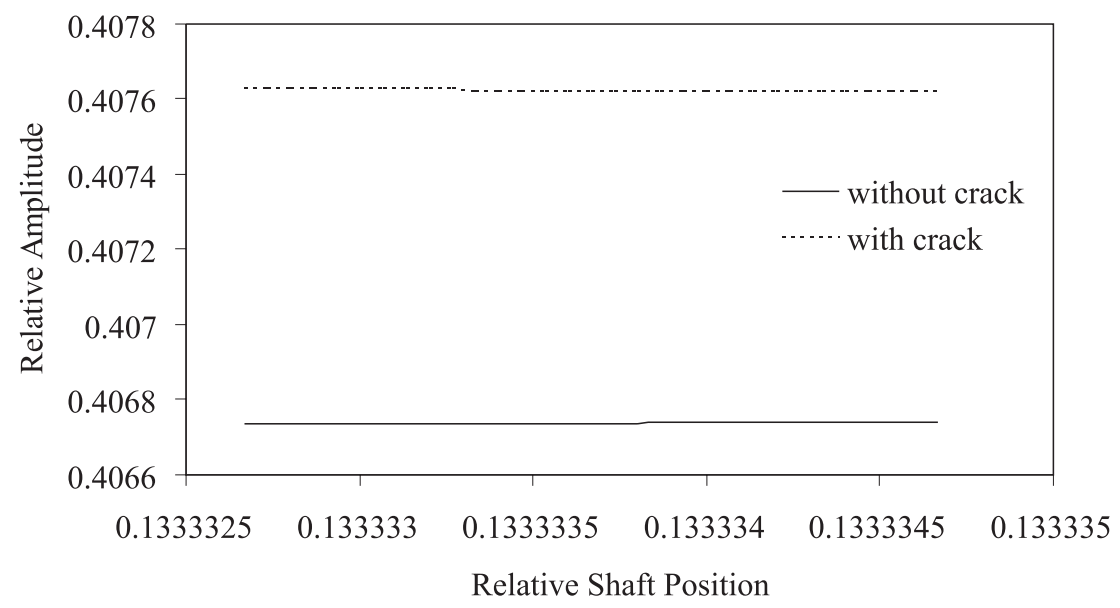

Figure 7. Magnified view of 1 st mode shape at second crack location $a_{1} / D=0.1, a_{2} / D=0.1$, $L_{1} / L=0.0667, L_{2} / L=0.1333, R_{1} / L=0.0667$.

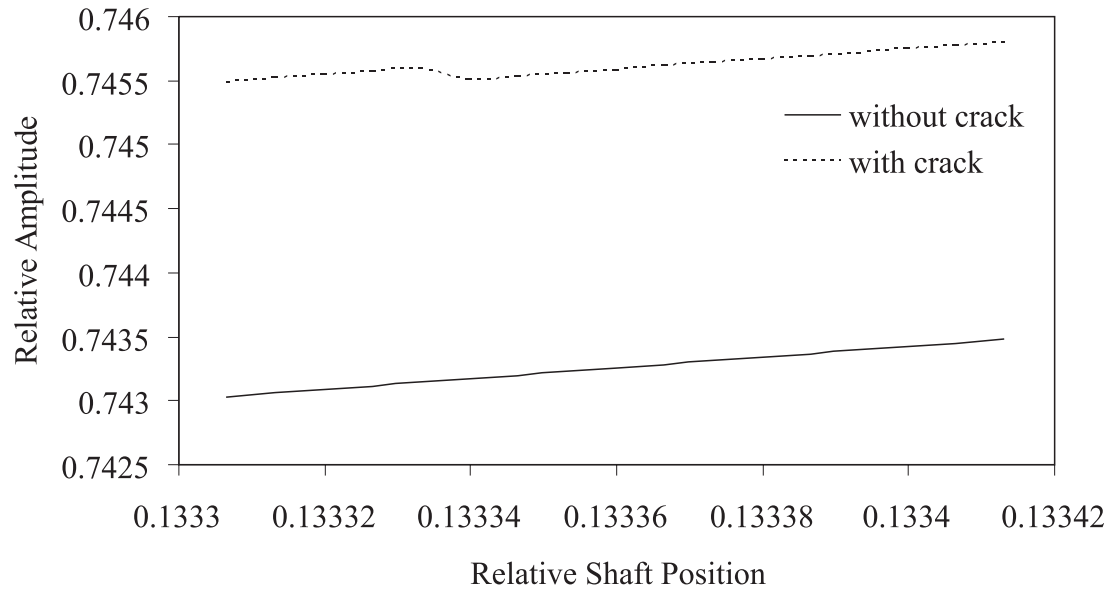

Figure 8. Magnified view of 2nd mode shape at second crack location $a_{1} / D=0.1, a_{2} / D=0.1$, $L_{1} / L=0.0667, L_{2} / L=0.1333, R_{1} / L=0.0667$.

The magnified views of mode shapes at the crack location presented in Figures 7 to 9 show abrupt changes in the mode shapes at the crack locations. The change in mode shapes between the uncracked shaft and a shaft with two cracks is shown in Figure 10, in which sudden changes in mode shapes at the crack locations $\left(L_{1} / L=0.0667, L_{2} / L=\right.$ 0.1333 ) can be seen. The position of the crack can be predicted from the deviations of the fundamental modes between the cracked and uncracked shafts. Furthermore, the crack size can be identified by the variation of the corresponding natural frequency, (shown in Figures 11, 12 and 13 for the first, second and third modes respectively). 


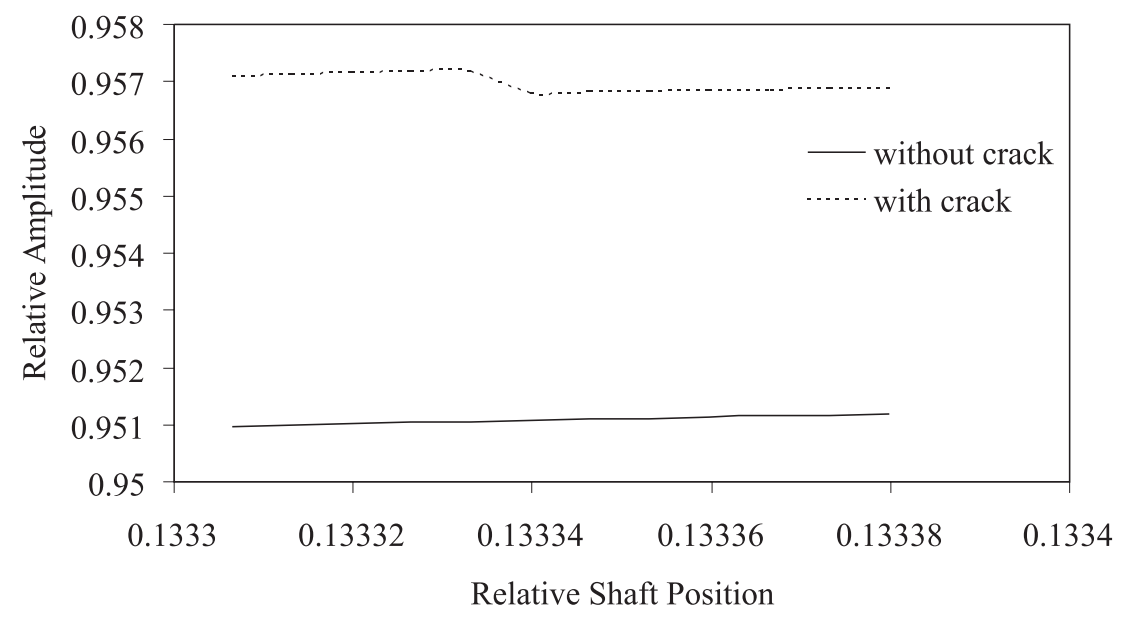

Figure 9. Magnified view of $3 r d$ mode shape at second crack location $a_{1} / D=0.1, a_{2} / D=0.1$, $L_{1} / L=0.0667, L_{2} / L=0.1333, R_{1} / L=0.0667$.

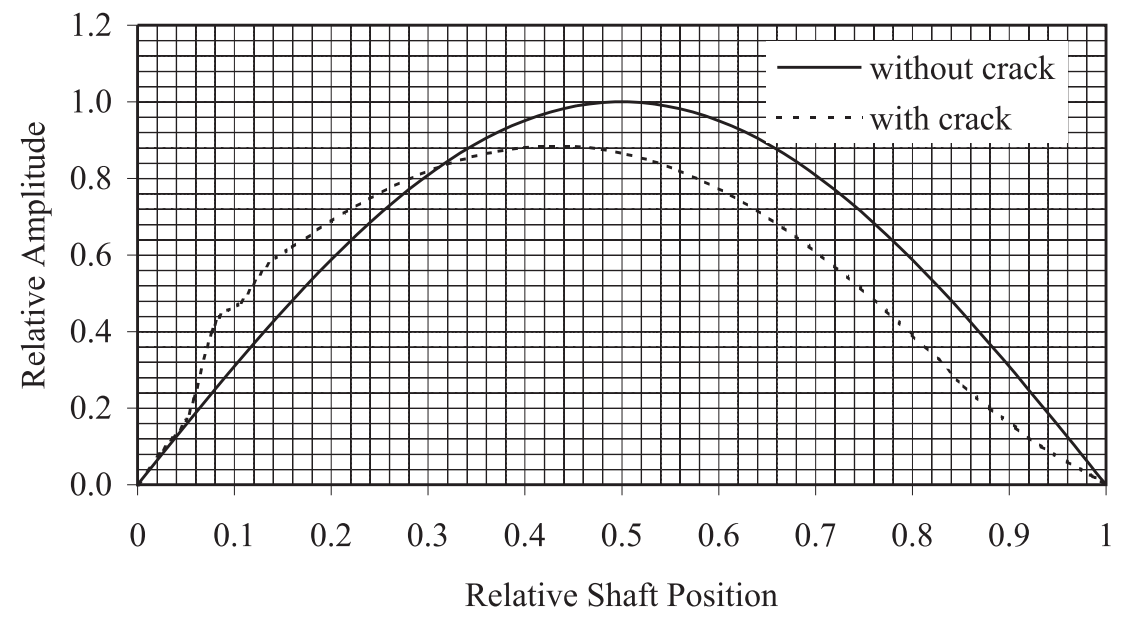

Figure 10. First mode of transverse vibration, $a_{1} / D=0.2, a_{2} / D=0.2, L_{1} / L=0.0667, L_{2} / L=0.1333$, $R_{1} / L=0.0667$.

The dynamic response of a continuously rotating cracked shaft $(L=1.0 \mathrm{~m}, r=$ $0.008 \mathrm{~m}$ ) was further analyzed as follows. The cracked shaft was allowed to rotate in a viscous fluid, and the amplitudes of rotor motion along the crack (44-direction) and perpendicular to the direction (55-direction) of crack studied. Figure 14 shows frequency against relative crack depth in the 44- and 55-directions. The frequency along the 55-direction decreases due to the decrease in stiffness compared to the 44-direction.

Comparisons for the dimensionless amplitude ratio in 44-direction (when it coincides with the $\mathrm{x}$ axis) and 55-direction (when it coincides with the $\mathrm{y}$ axis) against natural frequencies for various viscous mediums are shown in Figures 15 to 19. 


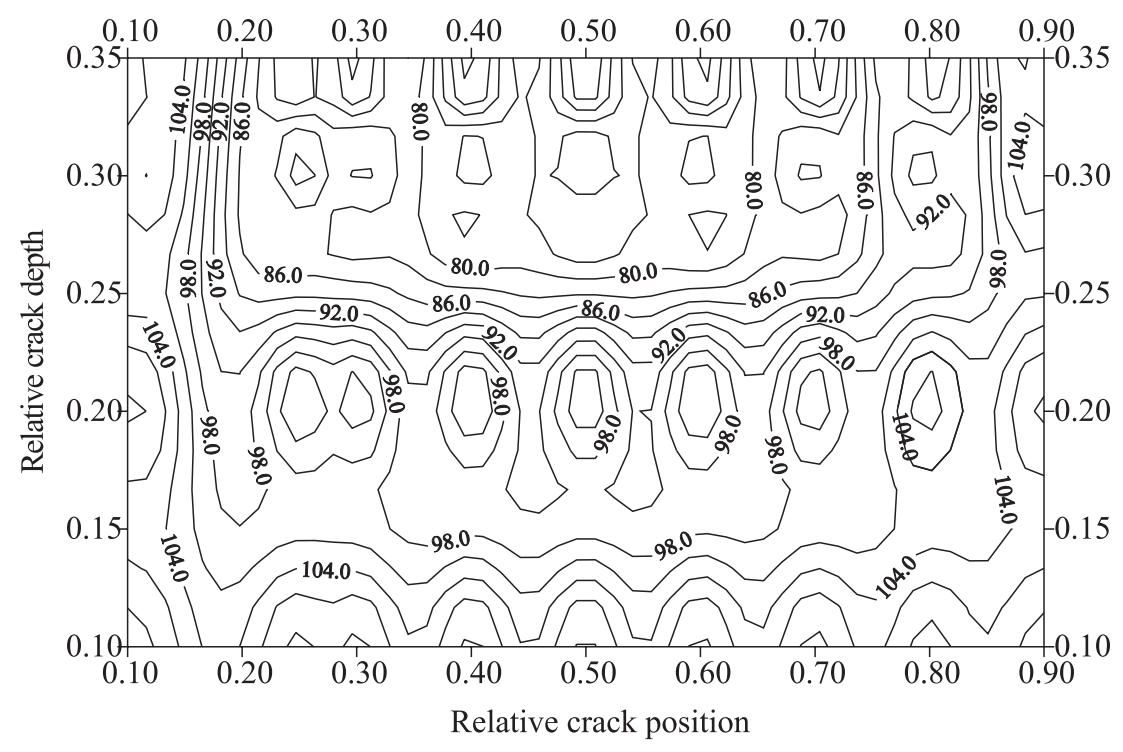

Figure 11. Contours of first fundamental frequencies of cracked shaft for various crack locations $\left(L_{2} / L\right)$ and depths $\left(a_{2} / D\right), a_{1} / D=0.1, L_{1} / L=0.1667$.

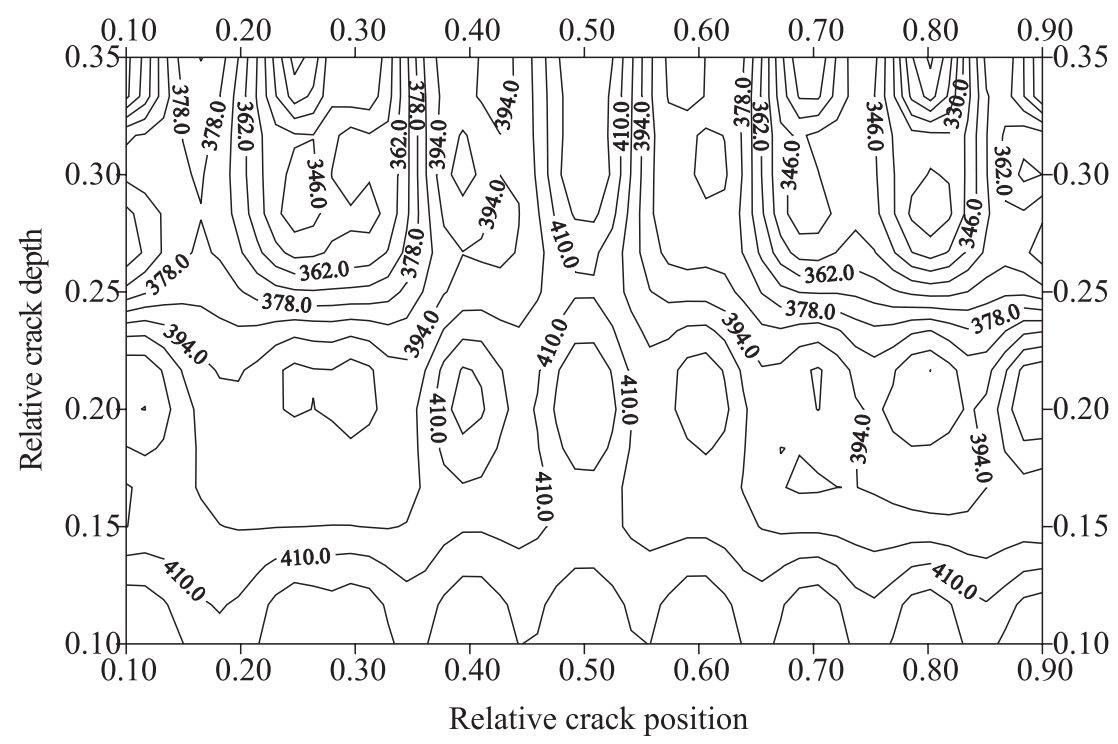

Figure 12. Contours of second fundamental frequencies of cracked shaft for various crack locations $\left(L_{2} / L\right)$ and depths $\left(a_{2} / D\right), a_{1} / D=0.1, L_{1} / L=0.1667$. 


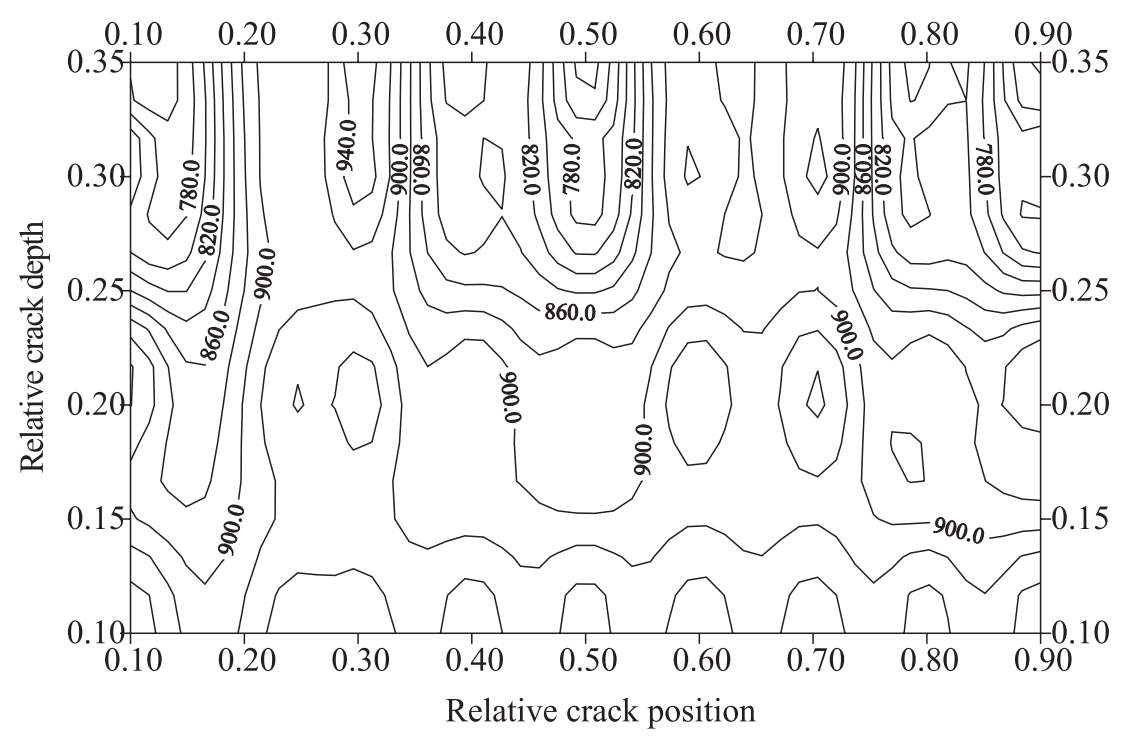

Figure 13. Contours of third fundamental frequencies of cracked shaft for various crack locations $\left(L_{2} / L\right)$ and depths $\left(a_{2} / D\right), a_{1} / D=0.1, L_{1} / L=0.1667$.

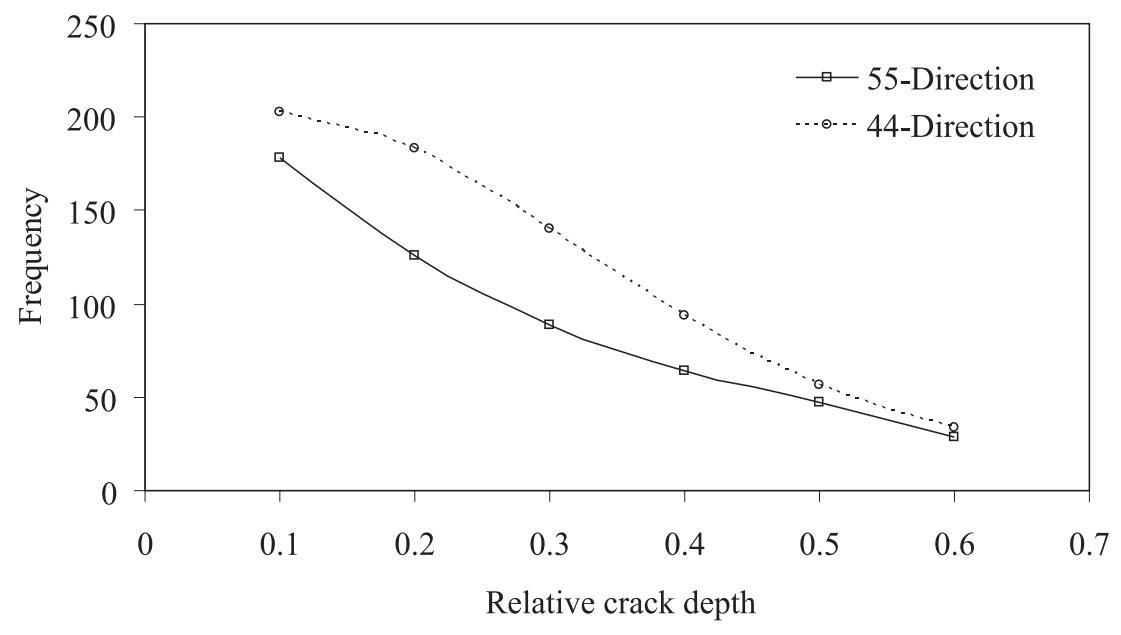

Figure 14. Frequency against relative crack depth of mild steel shaft $\left(R_{1}=0.008 \mathrm{~m}, L=1 \mathrm{~m}\right)$, $L_{1} / L=0.45, L_{2} / L=0.55$. 


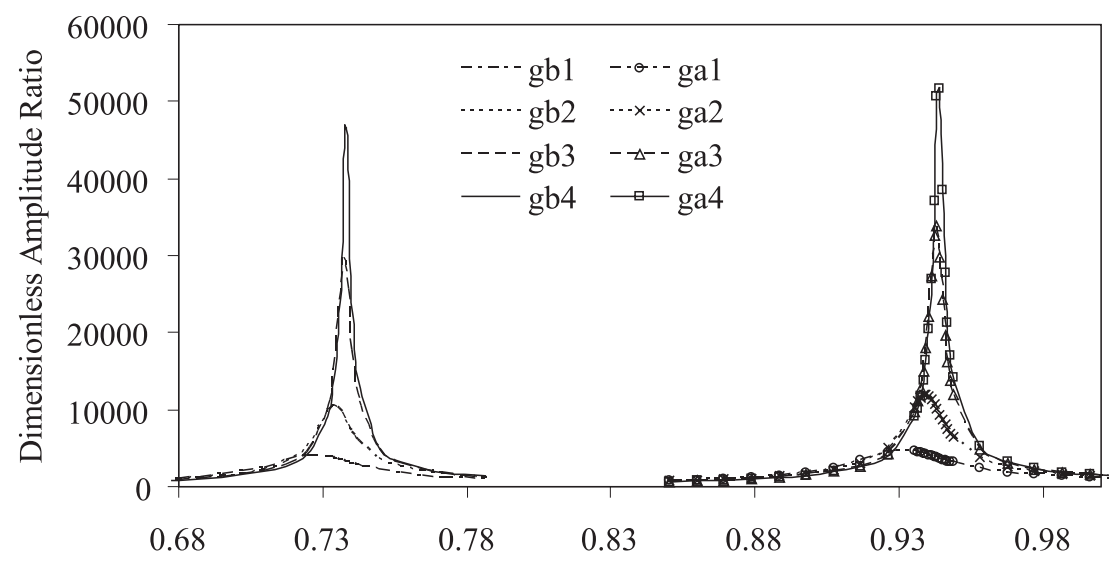

Frequency Ratio

Figure 15. Frequency ratio $\left(\omega / \omega_{0}\right)$ against dimensionless amplitude ratio $\left(\delta_{n}^{*} / \varepsilon^{*}\right)$, mild steel shaft specimen $\left(R_{1}=0.008 \mathrm{~m}, L=1 \mathrm{~m}\right), L_{1} / L=0.2, a_{1} / D=0.2$, ga1, gb1 $(v=2.3)$, ga2, gb2 $(v=0.427)$, ga3, gb3 $(v=0.0633)$, ga4, gb4 $(v=0.0284)$, ga $=44$-Direction, gb $=55$-Direction.

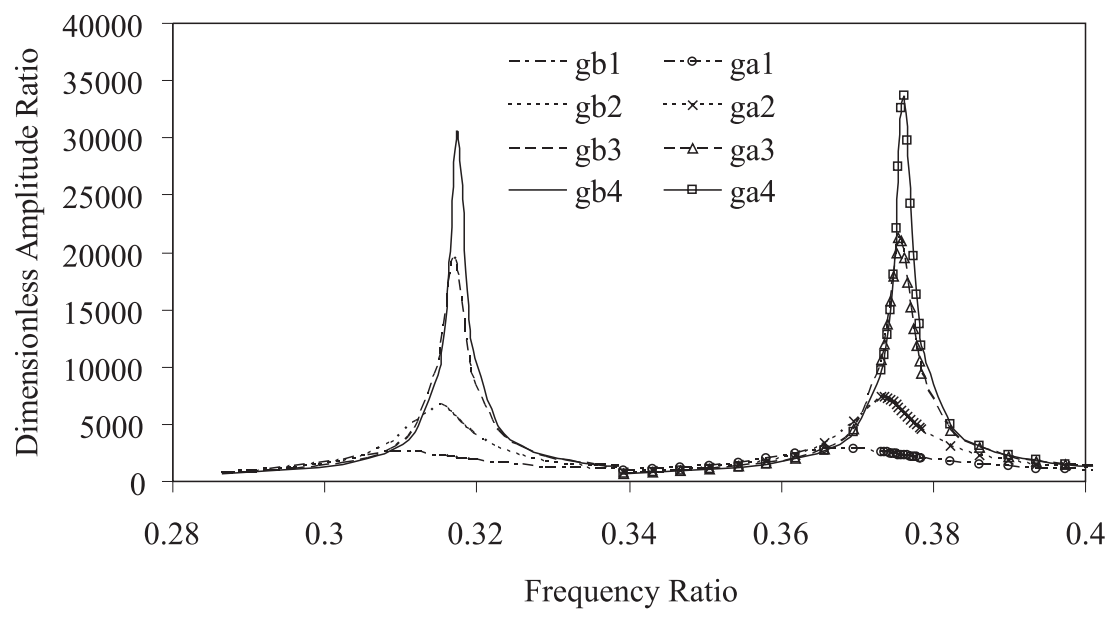

Figure 16. Frequency ratio $\left(\omega / \omega_{0}\right)$ against dimensionless amplitude ratio $\left(\delta_{n}^{*} / \varepsilon^{*}\right)$, mild steel shaft specimen $\left(R_{1}=0.008 \mathrm{~m}, L=1 \mathrm{~m}\right), L_{1} / L=0.5, a_{1} / D=0.5$, ga1, gb1 ( $\left.v=2.3\right)$, ga2, gb2 $(v=0.427), \mathrm{ga} 3, \mathrm{gb} 3(v=0.0633), \mathrm{ga} 4, \mathrm{gb} 4(v=0.0284), \mathrm{ga}=44-$ Direction, $\mathrm{gb}=55$-Direction.

For the numerical analysis of the rotor, the coefficients of viscosity are taken as 2.3 , $0.427,0.0633$ and 0.0284 Stokes. The relative crack depths considered are $0.2,0.3$ and 0.5 . For single crack rotor vibration analysis the crack location $\left(L_{1} / L\right)$ is selected such that $L_{1} / L=0.2$ and 0.5 (Figures 15 and 16, respectively). For two cracks, the relative crack locations $\left(L_{1} / L, L_{2} / L\right)$ are chosen as 0.45 and 0.55 respectively (Figures 17 to 19). 


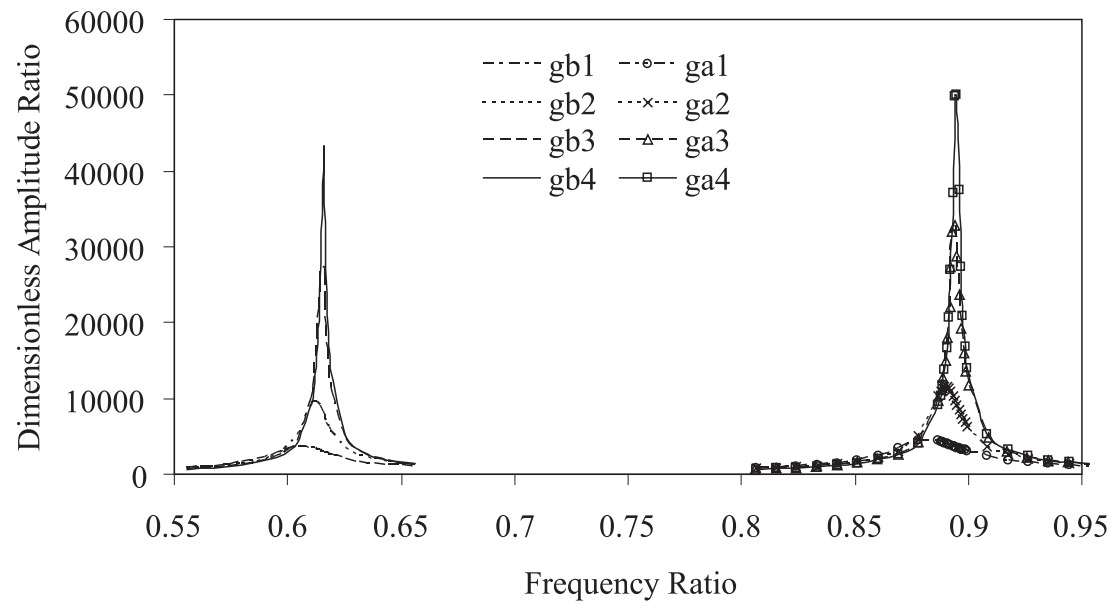

Figure 17. Frequency ratio $\left(\omega / \omega_{0}\right)$ against dimensionless amplitude ratio $\left(\delta_{n}^{*} / \varepsilon^{*}\right)$, mild steel shaft specimen $\left(R_{1}=0.008 \mathrm{~m}, L=1 \mathrm{~m}\right), L_{1} / L=0.45, L_{2} / L=0.55, a_{1} / D=0.2, a_{2} / D=0.2, \mathrm{ga} 1, \mathrm{gb} 1$ $(v=2.3)$, ga2, gb2 $(v=0.427)$, ga3, gb3 ( $v=0.0633)$, ga4, gb4 ( $v=0.0284)$, ga $=44-$ Direction, gb $=$ 55-Direction.

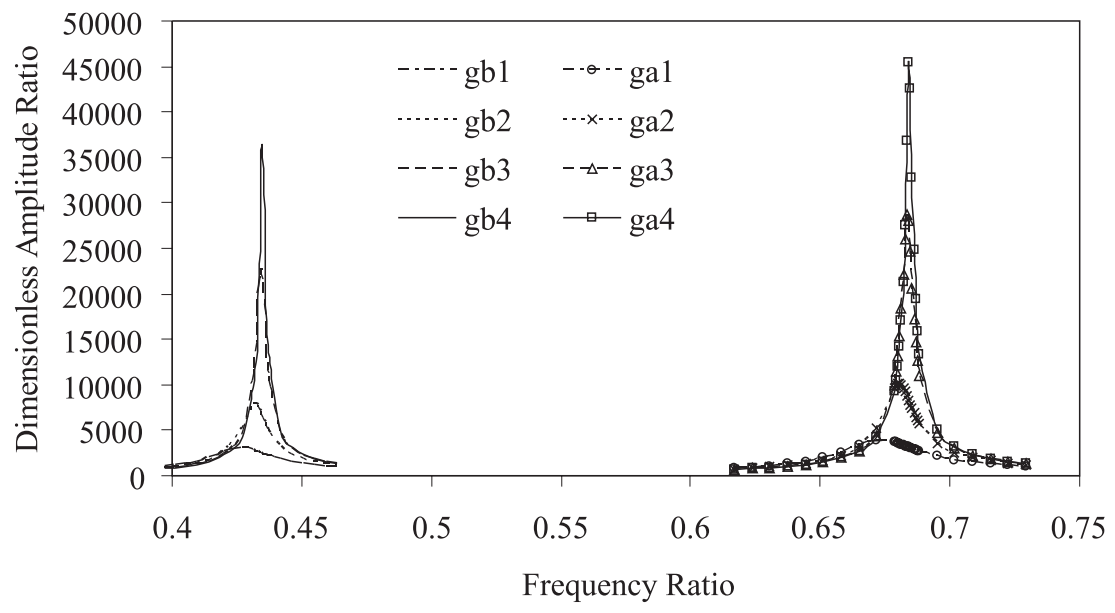

Figure 18. Frequency ratio $\left(\omega / \omega_{0}\right)$ against dimensionless amplitude ratio $\left(\delta_{n}^{*} / \varepsilon^{*}\right)$, mild steel shaft specimen $\left(R_{1}=0.008 \mathrm{~m}, L=1 \mathrm{~m}\right), L_{1} / L=0.45, L_{2} / L=0.55, a_{1} / D=0.3, a_{2} / D=0.3$, ga1, gb1 $(v=2.3)$, ga2, gb2 ( $v=0.427)$, ga3, gb3 $(v=0.0633)$, ga4, gb4 $(v=0.0284)$, ga $=44-D i r e c t i o n, g b=$ 55-Direction.

Comparisons of the amplitudes for uncracked and cracked rotors are shown in Figures 20 and 21. The amplitude of rotor motion decreases considerably for a deep crack $\left(a_{1} / D \geq 0.5\right)$.

To verify the authencity of the theory, the results (Appendix B) are compared with the experimental results (Figure 22(a)) of Waltson et al. (1964). A schematic view of the cantilever 


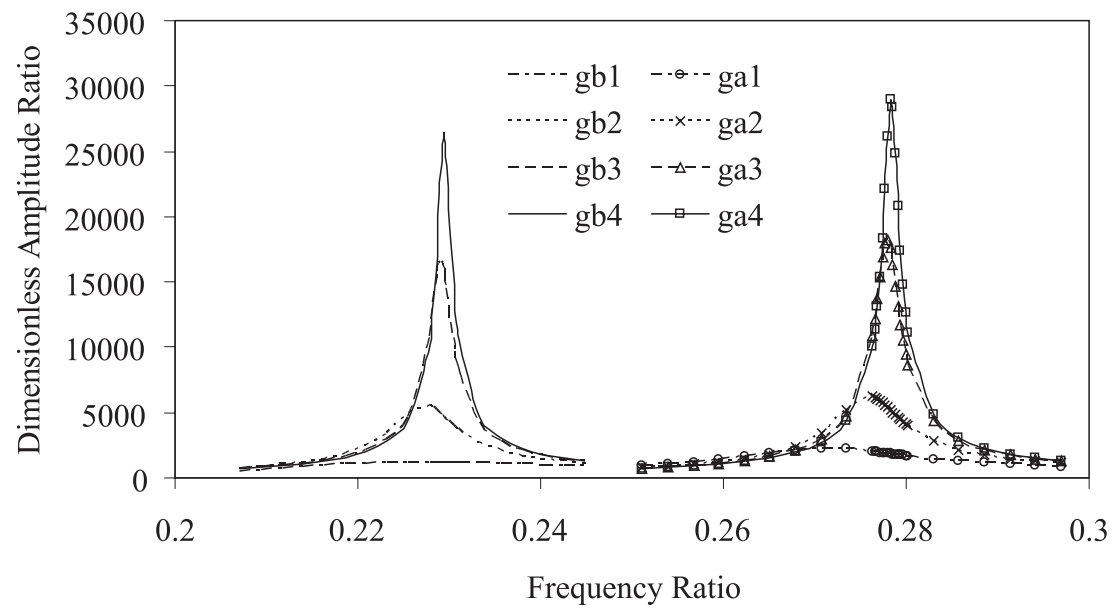

Figure 19. Frequency ratio $\left(\omega / \omega_{0}\right)$ against dimensionless amplitude ratio $\left(\delta_{n}^{*} / \varepsilon^{*}\right)$, mild steel shaft specimen $\left(R_{1}=0.008 \mathrm{~m}, L=1 \mathrm{~m}\right), L_{1} / L=0.45, L_{2} / L=0.55, a_{1} / D=0.5, a_{2} / D=0.5$, ga1, gb1 $(v=2.3)$, ga2, gb2 ( $v=0.427)$, ga3, gb3 $(v=0.0633)$, ga4, gb4 $(v=0.0284)$, ga $=44-$ Direction, gb $=$ 55-Direction.

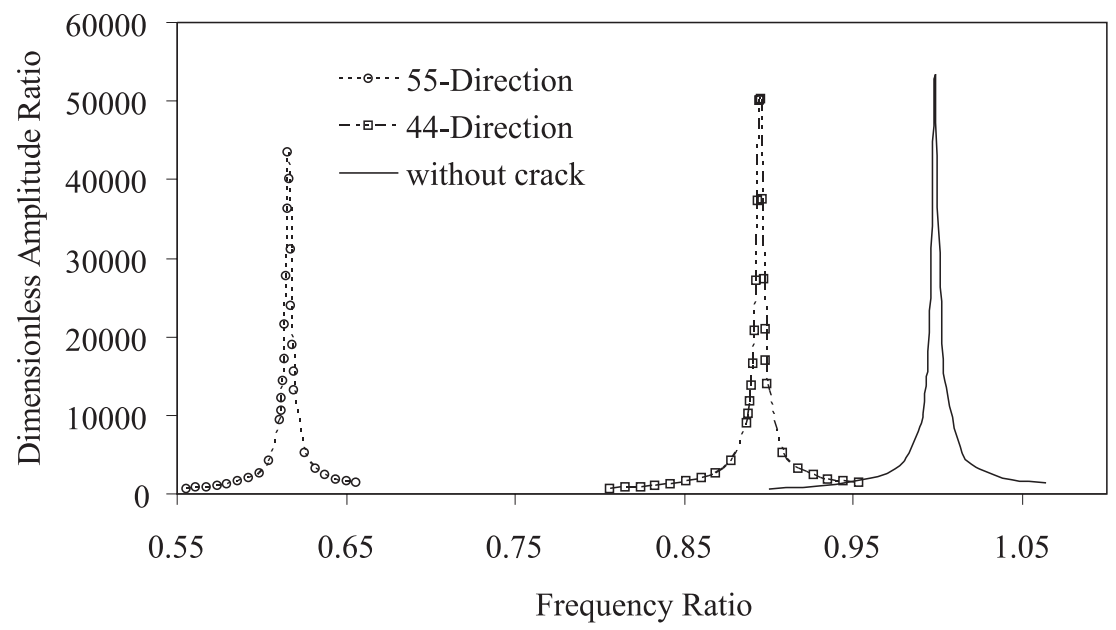

Figure 20. Frequency ratio $\left(\omega / \omega_{0}\right)$ against dimensionless amplitude ratio $\left(\delta_{n}^{*} / \varepsilon^{*}\right)$, mild steel shaft specimen $\left(R_{1}=0.008 \mathrm{~m}, L=1 \mathrm{~m}\right), L_{1} / L=0.45, L_{2} / L=0.55, a_{1} / D=0.2, a_{2} / D=0.2, v=0.0284$ Stoke.

rotor used in the analysis is shown in Figure 22(b), while Figure 23 shows a cross-section of the cracked rotor in the inertial frame. The Campbell diagrams for the cracked rotor are plotted and presented in Figures 24 (b) and (c). The bending frequencies for the cracked shaft change significantly compared to the uncracked one. 


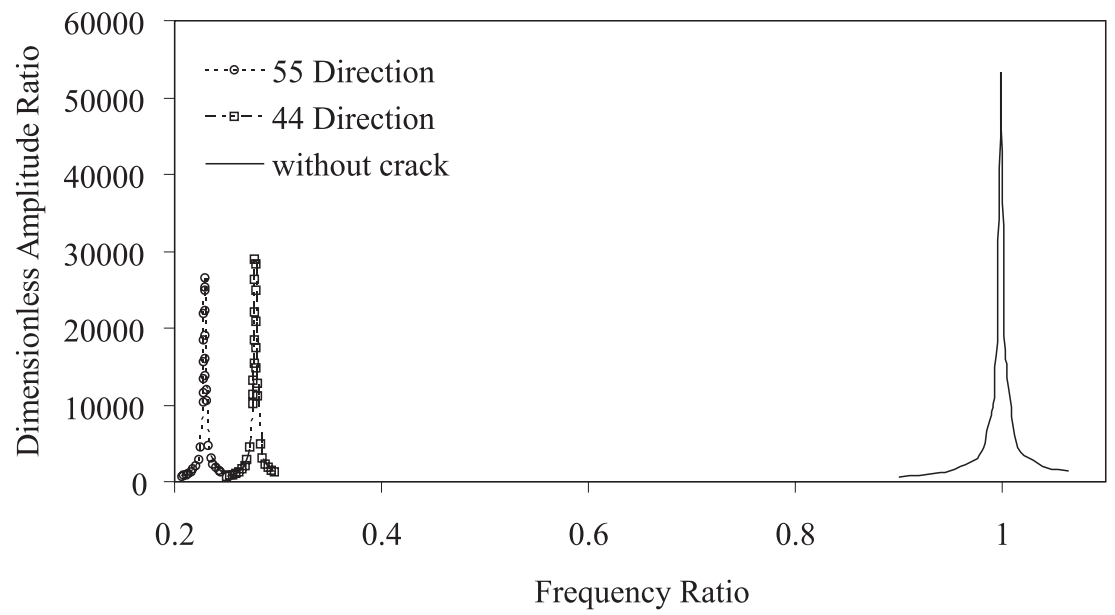

Figure 21. Frequency ratio $\left(\omega / \omega_{0}\right)$ against dimensionless amplitude ratio $\left(\delta_{n}^{*} / \varepsilon^{*}\right)$, mild steel shaft specimen $\left(R_{1}=0.008 \mathrm{~m}, L=1 \mathrm{~m}\right), L_{1} / L=0.45, L_{2} / L=0.55, a_{1} / D=0.5, a_{2} / D=0.5, v=0.0284$ Stoke.

Table1. Parameters in the theoretical analysis for finite fluid region.

\begin{tabular}{lc}
\hline Parameters & Properties \\
\hline Radius of shaft: $R_{1}$ & $0.012 \mathrm{~m}$ \\
Length of shaft: $L$ & $1.2 \mathrm{~m}$ \\
Density of shaft material: $\rho$ & $7860 \mathrm{Kg} / \mathrm{m}^{3}$ \\
Modulus of elasticity: $E$ & $200 \mathrm{Gpa}$ \\
Radius of disk & $0.04 \mathrm{~m}$ \\
Thickness of disk & $0.04 \mathrm{~m}$ \\
\hline
\end{tabular}

For experimental verification, the dimensions taken for the cantilever type rotor are:

Length of shaft $=0.762 \mathrm{~m}$

Radius of shaft $=0.00635 \mathrm{~m}$

Weight of disk $=1.3 \mathrm{~kg}$

Radius of disk $=0.054 \mathrm{~m}$

Liquid medium $=70 \%$ and $100 \%$ glycerin

It can be seen that the existing experimental results and the current numerical analysis are in good agreement.

The interaction between a cracked rotor with a disk and a finite fluid region in which it is rotating was then further investigated using different parameters as shown in the Table 1. Figures 25(a) and (b) show the effects of gap-ratio on the maximum whirling radius $\left(\delta_{\max }^{*} / \varepsilon^{*}\right)$, which increases with increasing gap-ratio. When the viscosity or relative crack depth increases, the maximum whirling radius decreases (Figures 25 (a) and (b). 

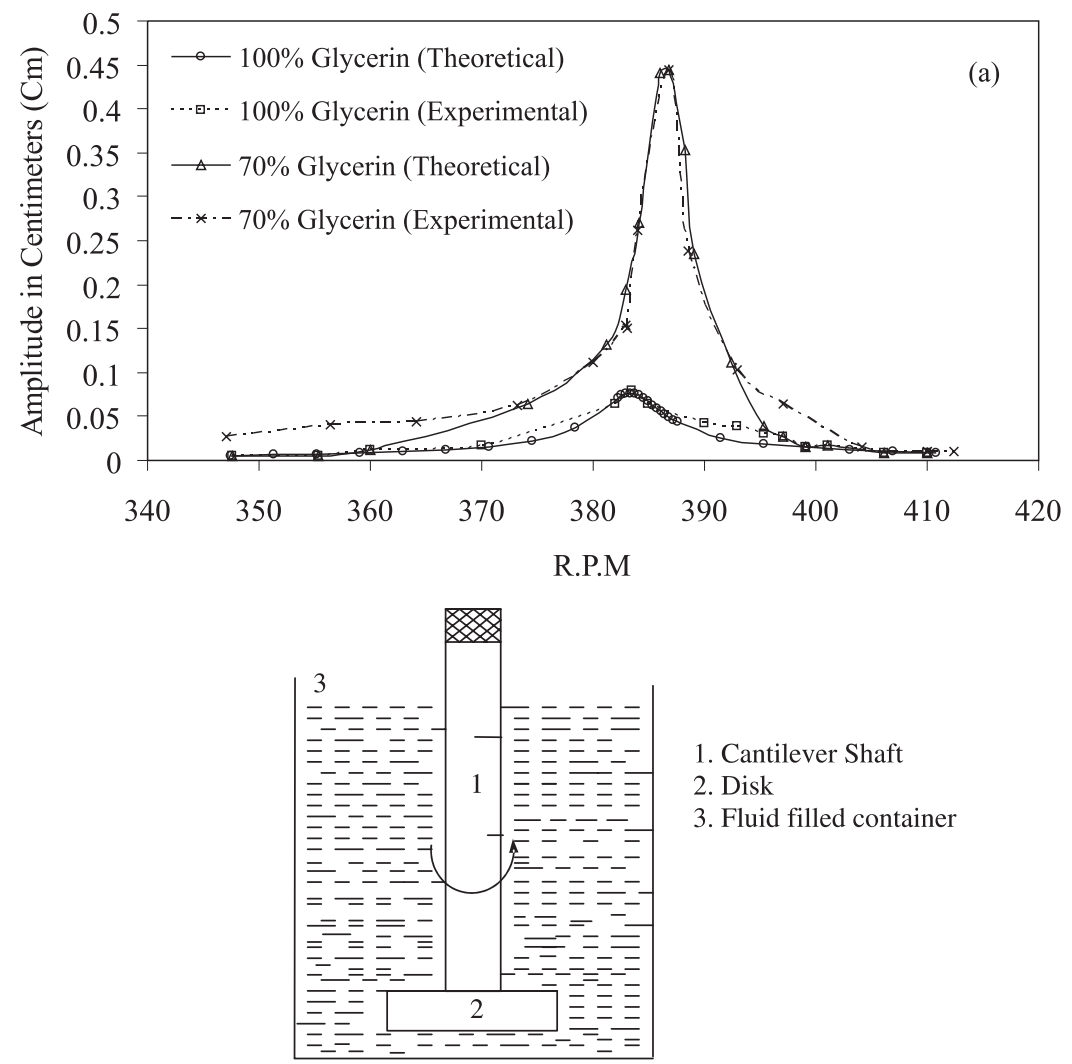

(b)

Figure 22. (a) Comparison of experimental result (Waltson) with theoretical result, (b) Cantilever rotor in fluid filled container.

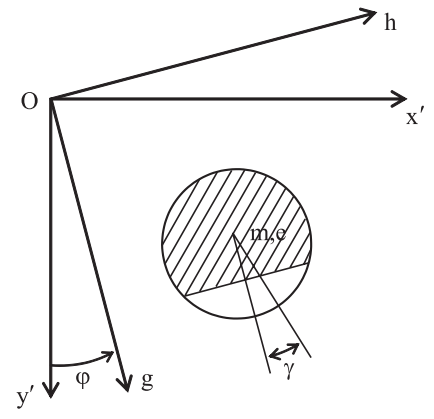

Figure 23. The cross-section of the cracked rotor in inertial frame. 


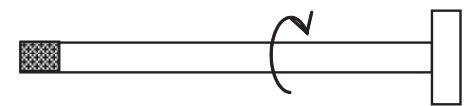

(a)

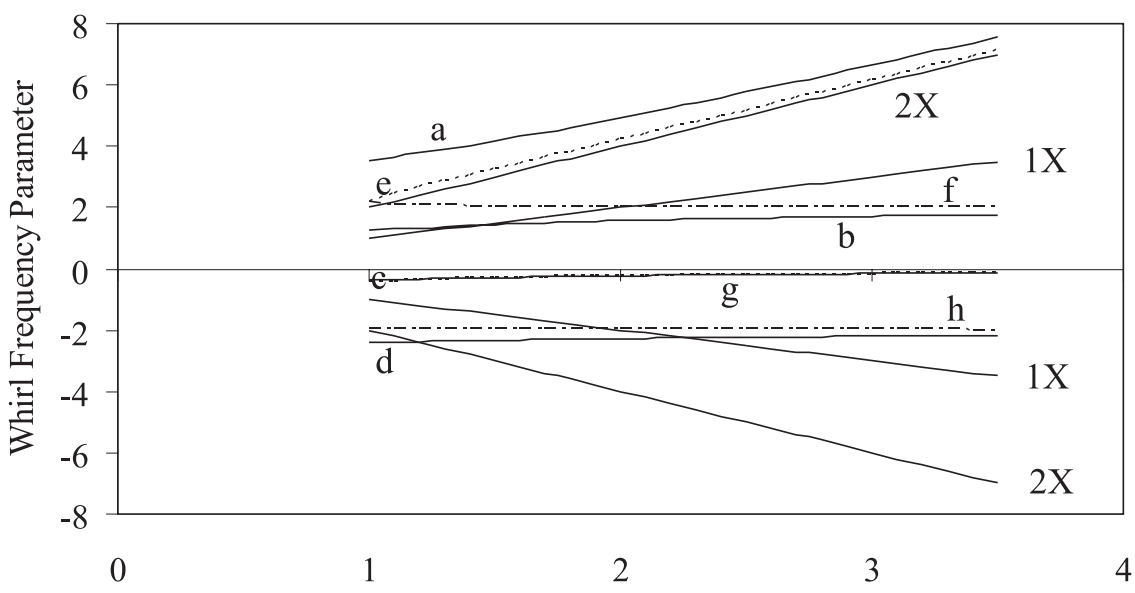

Rotational Speed Parameter

(b)

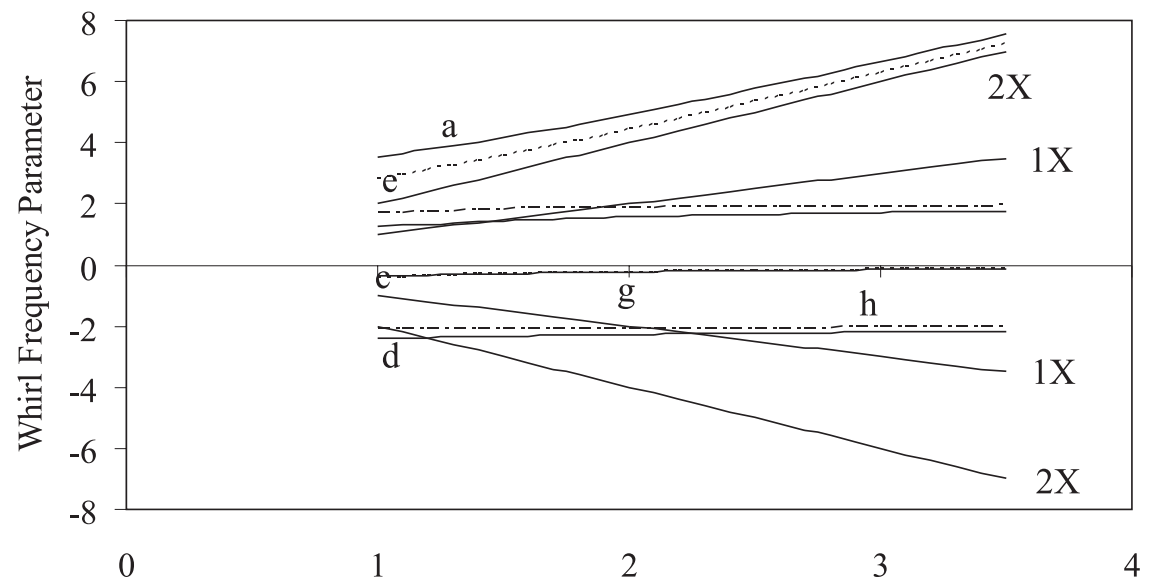

Rotational Speed Parameter

(c)

Figure 24. (a) Cantilever rotor with disk, (b) Speed against Frequency of a cracked rotor perpendicular to the crack (55) a, b, c, d = uncrack; e, f, g, h = with crack; $a_{1} / D=0.2, L_{1} / L=0.25 \mathrm{a}, \mathrm{b}, \mathrm{e}, \mathrm{f}=$ Forward whirl; c, d, g, h = Reverse whirl, (c) Speed against Frequency of a cracked rotor along the crack (44) $\mathrm{a}, \mathrm{b}, \mathrm{c}, \mathrm{d}=$ uncrack; e, f, $\mathrm{g}, \mathrm{h}=$ with crack; $a_{1} / D=0.2, L_{1} / L=0.25 \mathrm{a}, \mathrm{b}, \mathrm{e}, \mathrm{f}=$ Forward whirl; c,d,g, $\mathrm{h}=$ Reverse whirl. 


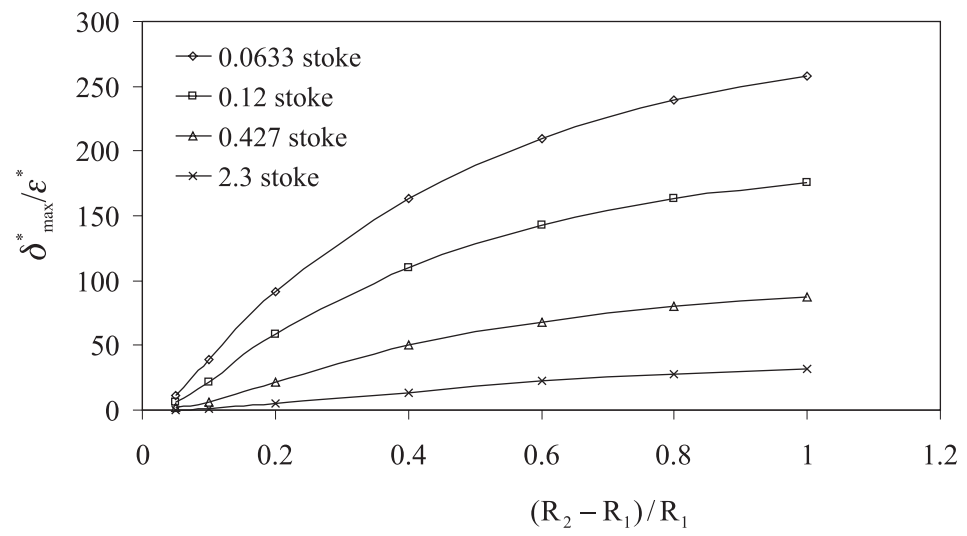

(a)

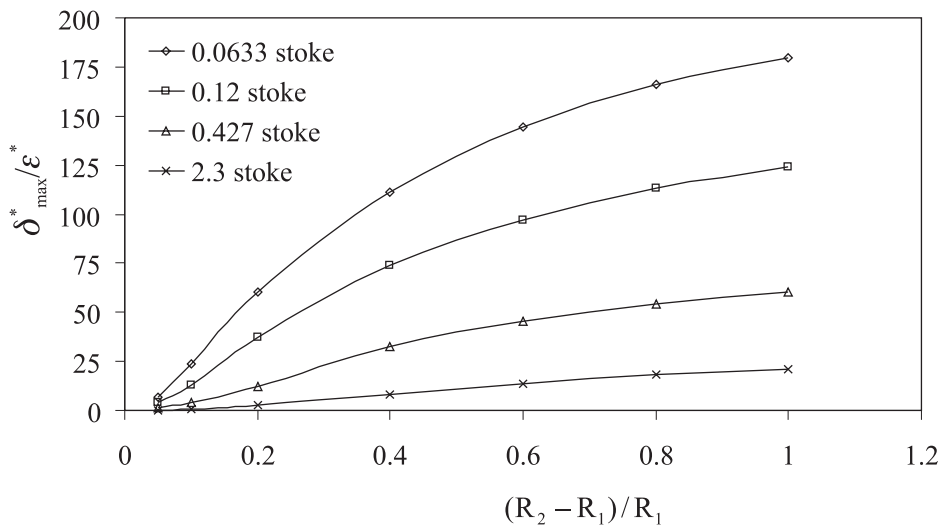

(b)

Figure 25. (a) Effect of gap-ratio and viscosity on maximum whirling radius of a rotor in circular cylindrical liquid region. $a_{1} / D=0.3, L_{1} / L=0.1667 R_{1}=0.012 \mathrm{~m}, L=1.2 \mathrm{~m}$, (b). Effect of gap-ratio and viscosity on maximum whirling radius of a rotor in circular cylindrical liquid region. $a_{1} / D=0.5, L_{1} / L=0.1667$, $R_{1}=0.012 \mathrm{~m}, L=1.2 \mathrm{~m}$.

The phase lags, calculated from equation 20(c), for uncracked and cracked rotors in infinitely extending fluid region are shown in Figures 26(a), (b) and (c). It was found that the phase lags along the 55- and 44- directions of a cracked rotor change considerably when compared to those of an uncracked one.

\section{CONCLUSIONS}

This paper presents a comprehensive dynamic analysis of a cracked rotor. In the numerical part of this study, the effect of crack depths and locations on modal properties of the rotor shaft is investigated. It was demonstrated that varying the crack location and depth of the cracks results in changes in the natural frequencies and amplitudes of vibration. Studies 

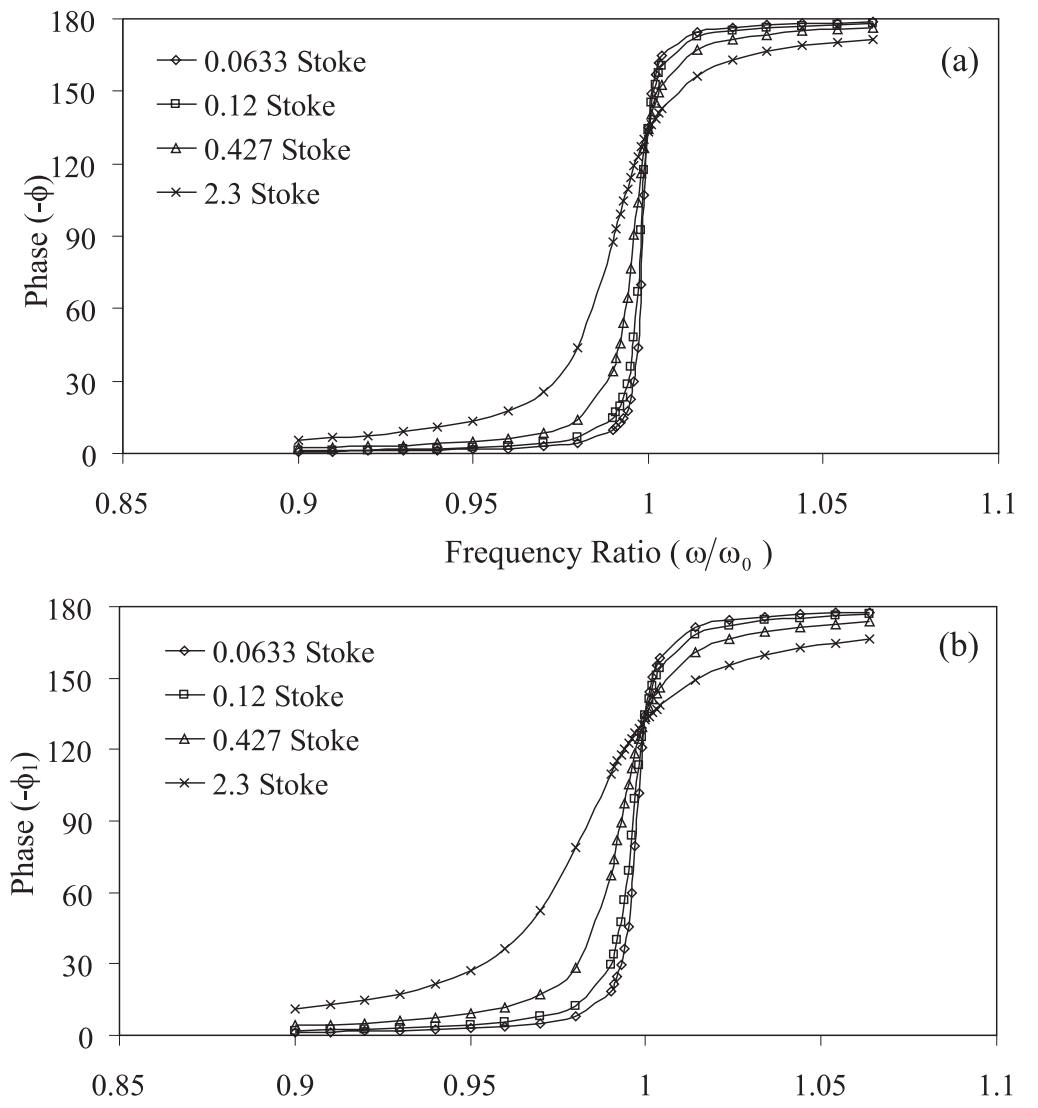

Frequency Ratio $\left(\omega / \omega_{0}\right)$

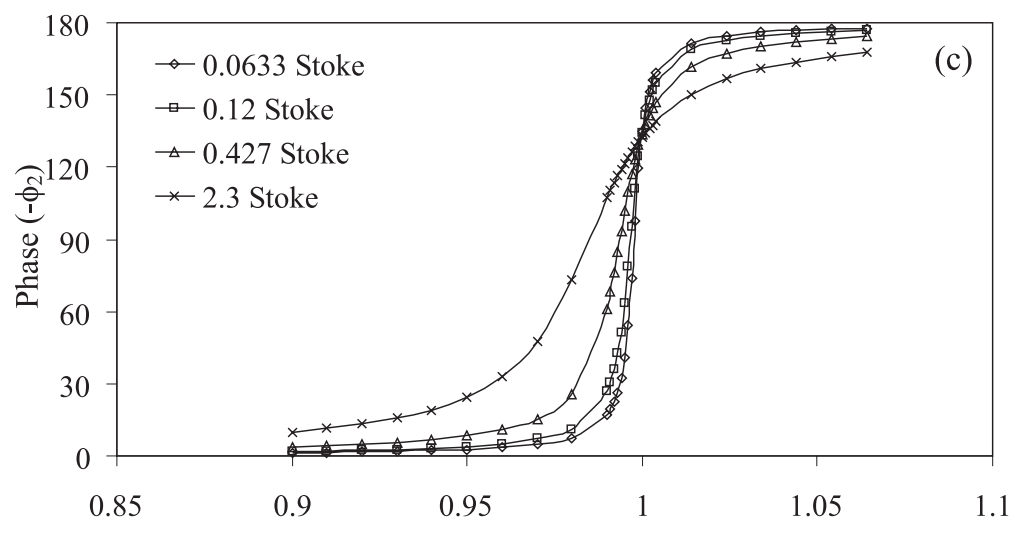

Frequency Ratio $\left(\omega / \omega_{0}\right)$

Figure 26. (a) Phase lag (degrees) for an uncracked rotor immersed in infinite fluid region for different viscosity, (b) Phase lag (degrees) in 55- Direction for a cracked rotor immersed in infinite fluid region for different viscosity, (c) Phase lag (degrees) in 44- Direction for a cracked rotor immersed in infinite fluid region for different viscosity. 
were also conducted on the dynamic behavior of a rotor in a viscous fluid medium. The amplitudes of vibrations are affected significantly by the presence of cracks and viscous medium. Additionally, the following conclusions can be drawn from the analysis:

1) When the depth of the cracks increases, the natural frequencies decrease as expected. This is because the reduction in stiffness is proportional to the depth of the crack. Due to the presence of a crack, a sharp change is found in the fundamental modes between the uncracked and cracked shafts. From these, one can determine the position of a transverse crack. From the contour plot, knowing the frequency and crack position, the crack depth can be evaluated.

2) From the dimensionless amplitude ratio versus frequency ratio plots, it can be seen that as the viscosity of the fluid increases, the critical speed decreases (due to the increase in critical mass $\mid m \operatorname{Re}(H)) \mid$ ), as does the amplitude (due to the increase in the damping factor $|-m \omega \operatorname{Im}(H)|)$.

3) The presence of a crack decreases the critical speed and, as the stiffness of the cracked shaft in the 55-direction is lower than the stiffness in the 44-direction, the critical speed in the 55-direction is lower than that in the 44- direction.

4) Due to the decrease in critical speed, the damping coefficient $|-m \omega \operatorname{Im}(H)|$ increases for which maximum dimensionless amplitude of the rotating cracked shaft is lower than that of the uncracked shaft. The results are shown in Figures 20 and 21. External damping has a more significant effect in reducing the amplitude of vibration than in changing the resonance speed, but the virtual mass effect reduces the resonance speed considerably more than it reduces the amplitude of vibration.

5) The phase lag of the rotor changes due to the presence of a crack and viscous fluid.

It is believed that this research will provide a benchmark for the vibration analysis of cracked shafts and structures. Furthermore, the results obtained from the current analysis can be utilized for vibration monitoring of (a) high-speed boring machines, (b) high-speed rotors in centrifuges (prone to fatigue), (c) Turbine rotors, (d) rotors used for drilling oil from the sea bed and (e) rotors of ship, and also for preventing failure of rotors used in machines subjected to various environmental conditions.

\section{APPENDIX A}

\section{Analysis of the Vibration Characteristic of the Cracked Shaft}

A simply supported shaft of length $L$ and radius $R_{1}$, with cracks of depths $a_{1}$ and $a_{2}$ at distances $L_{1}$ and $L_{2}$ from the left-hand end is shown in Figure 2. A crack width of $2 \mathrm{~b}$ is considered on the shaft. $\theta_{1}(z, t), \theta_{2}(z, t)$ and $\theta_{3}(z, t)$ are the deflections caused by torsion vibrations in the sections before and after the cracks. Similarly, $Y_{1}(z, t), Y_{2}(z, t)$, and $Y_{3}(z, t)$ are the deflections from bending vibrations and $\xi_{1}(z, t), \xi_{2}(z, t)$, and $\xi_{3}(z, t)$ are the slopes of the deflection curves for the same sections. The normal functions can be written in dimensionless form as 


$$
\begin{aligned}
\bar{\theta}_{i=1,3}(\bar{z}) & =A_{i=1,3} \cos \left(\bar{k}_{\theta} \bar{z}\right)+B_{i=1,3} \sin \left(\bar{k}_{\theta} \bar{z}\right) \\
\bar{Y}_{i=1,3}(\bar{z}) & =a_{i=1,3} \sin \left(\lambda_{1} \bar{z}\right)+b_{i=1,3} \cos \left(\lambda_{1} \bar{z}\right) \\
& +c_{i=1,3} \sinh \left(\lambda_{2} \bar{z}\right)+d_{i=1,3} \cosh \left(\lambda_{2} \bar{z}\right) \\
\bar{\xi}_{i=1,3}(\bar{z}) & =a_{i=1,3} \Lambda_{1} \cos \left(\lambda_{1} \bar{z}\right)-b_{i=1,3} \Lambda_{1} \sin \left(\lambda_{1} \bar{z}\right) \\
& +c_{i=1,3} \Lambda_{2} \cosh \left(\lambda_{2} \bar{z}\right)+d_{i=1,3} \Lambda_{2} \sinh \left(\lambda_{2} \bar{z}\right)
\end{aligned}
$$

where, $\bar{z}=\frac{z}{L}, \bar{\theta}_{i}=\frac{\theta_{i}}{L}, \bar{Y}_{i}=\frac{Y_{i}}{L}, \bar{\xi}_{i}=\frac{\xi_{i}}{L}$ for $i=1,2,3$

$$
\begin{aligned}
& \bar{k}_{\theta}=\frac{\omega L}{C_{u}}, \quad C_{u}=\left(\frac{G}{\rho_{1}}\right)^{1 / 2}, \quad \mu_{s}=A_{s} \rho_{s}, \quad \psi^{2}=\frac{\mu_{s} L^{4} \omega^{2}}{E I}\left(1-\frac{\rho_{s} I \omega^{2}}{k k G A_{s}}\right) \\
& \lambda_{0}^{2}=\frac{\mu_{s} L^{2} \omega^{2}}{A_{s} E}\left(1+\frac{E}{k k G}\right), \quad \lambda_{1}^{2}=\left(\sqrt{\left.\psi^{4}+\frac{\lambda_{0}^{4}}{4}+\frac{\lambda_{0}^{2}}{2}\right),}\right. \\
& \lambda_{2}^{2}=\left(\sqrt{\psi^{4}+\frac{\lambda_{0}^{4}}{4}-\frac{\lambda_{0}^{2}}{2}}\right), \quad \lambda_{3}^{2}=\frac{\omega^{2}}{\left[k k G A_{s} /\left(\mu_{s} L^{2}\right)\right]}, \\
& \Lambda_{1}=\frac{\lambda_{1}-\lambda_{3}}{\lambda_{1}}, \quad \Lambda_{2}=\frac{\lambda_{2}+\lambda_{3}}{\lambda_{1}}
\end{aligned}
$$

Applying the boundary conditions to the normal function equations (equations (22a) to (22c)), the fundamental critical speed in the 55- and 44-directions can be calculated from [AQ](2004).

\section{Crack Modeling}

The crack model as shown in Figure 2 comprises all of the loading conditions $\left(P_{i}, i=1\right.$ to 6). The compliance matrix elements $C_{i j}$ at crack location can be written as;

$$
C_{i j}=\frac{\partial^{2}}{\partial P_{i} \partial P_{j}} \int_{-b}^{+b} \int_{a_{2}}^{a_{3}} J(a) \mathrm{d} y \mathrm{~d} x
$$

Where $J(a)=$ strain energy density function; $P_{i}, i=1$ to 6

Axial force $(i=1)$

Shear force $(i=2,3)$

Bending moment $(i=4,5)$

Torsional moment $(i=6)$

$a_{1}=$ depth of crack

$a_{2}=R_{1}-a_{1}, a_{3}=h_{1} / 2$ 
The global stiffness matrix $\left[K_{g}\right]$ can be written as

$$
\left[K_{g}\right]=\left[\begin{array}{lll}
K_{55} & K_{54} & K_{51} \\
K_{45} & K_{44} & K_{41} \\
K_{15} & K_{14} & K_{11}
\end{array}\right]=\left[\left[G_{1}\right]\left[C_{c r}\right]\left[G_{2}\right]+\left[C_{s}\right]\right]^{-1}
$$

where $\left[G_{1}\right]=\operatorname{diag}[L / 2, L / 2,1],\left[G_{2}\right]=[L / 4, L / 4,1]$,

$$
\left[C_{s}\right]=\operatorname{diag}\left[\frac{L^{3}}{48 E I}, \frac{L^{3}}{48 E I}, \frac{L}{E A_{s}}\right], \quad\left[C_{c r}\right]=\frac{1}{F_{0}}\left[\begin{array}{ccc}
\bar{C}_{55} / R_{1} & \bar{C}_{54} / R_{1} & \bar{C}_{51} \\
\bar{C}_{45} / R_{1} & \bar{C}_{44} / R_{1} & \bar{C}_{41} \\
\bar{C}_{15} & \bar{C}_{14} & \bar{C}_{11} / R_{1}
\end{array}\right]
$$

where $F_{0}=A_{s} E /\left(1-v_{1}^{2}\right)$ and diag $[\ldots]=$ diagonal matrix.

\section{Stiffness Calculation in Inertial Frame}

The stiffness matrix in the inertial frame can be obtained from the stiffness matrix in the rotational frame by the transformation matrix method. The stiffness matrix in the rotational frame is as follows:

$$
\left[\begin{array}{cc}
k_{g} & 0 \\
0 & k_{h}
\end{array}\right]=\left[\begin{array}{cc}
k_{u} & 0 \\
0 & k_{h}
\end{array}\right]-\Theta\left[\begin{array}{cc}
\Delta k_{g} & 0 \\
0 & 0
\end{array}\right]
$$

where $k_{g}\left(=K_{55}\right)$ is the stiffness in the g-axis direction, $k_{h}\left(=K_{44}\right)$ is the stiffness in the h-axis direction, $k_{u}$ is the stiffness of the uncracked rotor, and $\Delta k_{g}$ is the stiffness variation in the $\mathrm{g}$-axis direction.

The value $\Theta$ for the opening and closing of the crack can be written as

$$
\Theta=\left\{\begin{array}{lll}
1 & 2 n \pi \leq \theta_{1}<(2 n+1 / 2) \pi & \text { the crack is open } \\
0 & (2 n+1 / 2) \pi \leq \theta_{1}<(2 n+3 / 2) \pi & \text { the crack is closed } \\
1 & (2 n+3 / 2) \pi \leq \theta_{1}<(2 n+2) \pi & \text { the crack is open }
\end{array}\right.
$$

where $n=0,1,2, \ldots, \theta_{1}=\frac{1}{2} a^{\prime} t^{2}+\omega_{0}^{\prime} t+\gamma, a^{\prime}$ is the angular acceleration, $\omega_{0}^{\prime}$ is the initial angular speed, and $\gamma$ is the angle of unbalance with respect to the g-axis.

By Fourier transform, equation (24) can be written as

$$
\Theta=\frac{1}{2}+\frac{2}{\pi} \cos \theta_{1}-\frac{2}{3 \pi} \cos 3 \theta_{1}+\frac{2}{5 \pi} \cos 5 \theta_{1}-\cdots
$$

From Figure 22, the transformation matrix is 


$$
T=\left[\begin{array}{cc}
\cos \varphi & \sin \varphi \\
-\sin \varphi & \cos \varphi
\end{array}\right],
$$

where $\varphi=\frac{1}{2} a^{\prime} t^{2}+\omega_{0}^{\prime} t$.

The stiffness matrix in the inertial frame can be written as

$$
\left[\begin{array}{cc}
k_{x^{\prime}} & k_{x^{\prime} y^{\prime}} \\
k_{x^{\prime} y^{\prime}} & k_{y^{\prime}}
\end{array}\right]=T^{-1}\left[\begin{array}{cc}
k_{g} & 0 \\
0 & k_{h}
\end{array}\right] T
$$

\section{APPENDIX B}

\section{Vibration Analysis of Cantilever Rotor Rotating in a Viscous Fluid}

The cantilever spinning shaft with the disk at the free end is considered to be a lumped system with the mass of the disk and equivalent mass of the shaft lumped at its free end. For calculating the dynamic response of the above system, the mass of the disk is represented by $M_{s 1}$, which is attached to the free end of the shaft, and the mass of the shaft is represented by $M_{s 2}$. An equivalent lumped mass to the rotating shaft is given by $K_{s} / \omega_{1}^{2}$, where $K_{s}$ is the shaft stiffness and $\omega_{1}$ is the fundamental natural angular frequency of the shaft. The total lumped mass of the rotor system becomes

$$
M_{s}=M_{s 1}+\alpha_{e q} M_{s 2}
$$

where

$$
\alpha_{e q}=\frac{K_{s}}{\omega_{1}^{2} M_{s 2}}
$$

The equation of motion of an equivalent single-degree-of-freedom system of the whirling rotor in fluid is given by

$$
\begin{aligned}
& M_{s} \frac{\mathrm{d}^{2}(x+\varepsilon \cos \omega t)}{\mathrm{d} t^{2}}+K_{s} x=F_{x} \\
& M_{s} \frac{\mathrm{d}^{2}(y+\varepsilon \sin \omega t)}{\mathrm{d} t^{2}}+K_{s} y=F_{y}
\end{aligned}
$$

where $\varepsilon$ denotes the distance between the shaft center and the center of gravity of the rotor, and any internal damping of the shaft is neglected. In this case the fluid forces $F_{x}$ and $F_{y}$ given by equation (16) are rewritten into

$$
F_{x}=-M \operatorname{Re}(H) \frac{\mathrm{d}^{2} x}{\mathrm{~d} t^{2}}+M \omega \operatorname{Im}(H) \frac{\mathrm{d} x}{\mathrm{~d} t}
$$




$$
F_{y}=-M \operatorname{Re}(H) \frac{\mathrm{d}^{2} y}{\mathrm{~d} t^{2}}+M \omega \operatorname{Im}(H) \frac{\mathrm{d} y}{\mathrm{~d} t}
$$

where the virtual mass $M \operatorname{Re}(H)$ and the damping coefficient $M \omega \operatorname{Im}(H)$ are given as

$$
\begin{aligned}
M \operatorname{Re}(H) & =M_{1} \operatorname{Re}\left(H_{1}\right)+\alpha_{e q} M_{2} \operatorname{Re}\left(H_{2}\right) \\
M \omega \operatorname{Im}(H) & =M_{1} \omega \operatorname{Im}\left(H_{1}\right)+\alpha_{e q} M_{2} \omega \operatorname{Im}\left(H_{2}\right)
\end{aligned}
$$

where $M_{1}$ and $M_{2}$ denote the fluid masses displaced by the disk and shaft respectively, $M_{1} \operatorname{Re}\left(H_{1}\right)$ and $M_{1} \omega \operatorname{Im}\left(H_{1}\right)$ mean the virtual mass and the damping coefficient of the disk respectively, and $M_{2} \operatorname{Re}\left(H_{2}\right)$ and $M_{2} \omega \operatorname{Im}\left(H_{2}\right)$ are those of the shaft. A lumped fluid mass $M$ displaced by the rotor can be expressed in the same way as the total lumped mass of rotor $M_{s}$; that is

$$
M=M_{1}+\alpha_{e q} M_{2}
$$

From equations (29) and (30), we get the equations of motion for the rotor in fluid:

$$
\begin{aligned}
& \left(M_{s}+M \operatorname{Re}(H)\right) \frac{\mathrm{d}^{2} x}{\mathrm{~d} t^{2}}-M \omega \operatorname{Im}(H) \frac{\mathrm{d} x}{\mathrm{~d} t}+K_{s} x=M_{s} \varepsilon \omega^{2} \cos \omega t \\
& \left(M_{s}+M \operatorname{Re}(H)\right) \frac{\mathrm{d}^{2} y}{\mathrm{~d} t^{2}}-M \omega \operatorname{Im}(H) \frac{\mathrm{d} y}{\mathrm{~d} t}+K_{s} y=M_{s} \varepsilon \omega^{2} \sin \omega t
\end{aligned}
$$

Equation (31) can be written in dimensionless form as

$$
\begin{aligned}
& \left(1+M^{*} \operatorname{Re}(H)\right) \frac{\mathrm{d}^{2} \xi}{\mathrm{d} \tau^{2}}-M^{*} \omega^{*} \operatorname{Im}(H) \frac{\mathrm{d} \xi}{\mathrm{d} \tau}+\xi=\varepsilon^{*}\left(\omega^{*}\right)^{2} \cos \left(\omega^{*} \tau\right) \\
& \left(1+M^{*} \operatorname{Re}(H)\right) \frac{\mathrm{d}^{2} \eta}{\mathrm{d} \tau^{2}}-M^{*} \omega^{*} \operatorname{Im}(H) \frac{\mathrm{d} \eta}{\mathrm{d} \tau}+\eta=\varepsilon^{*}\left(\omega^{*}\right)^{2} \sin \left(\omega^{*} \tau\right)
\end{aligned}
$$

where $\xi=\frac{x}{R_{1}}, \eta=\frac{y}{R_{1}}, \omega^{*}=\frac{\omega}{\omega_{0}}, \varepsilon^{*}=\frac{\varepsilon}{R_{1}}, M^{*}=\frac{M}{M_{s}}, \tau=\omega_{0} t, \omega_{0}=\sqrt{\frac{K_{s}}{M_{s}}}$

The steady state solution of the above equation can be obtained in dimensionless form as

$$
\xi=\delta^{*} \cos \left(\omega^{*} \tau-\phi\right)
$$

where

$$
\begin{aligned}
\delta^{*} & =\frac{A}{\sqrt{\left(K-\omega^{* 2}\right)^{2}+\left(C \omega^{*}\right)^{2}}}, \quad \phi=\tan ^{-1}\left(\frac{C \omega^{*}}{K-\omega^{* 2}}\right), \quad C=\frac{M^{*} \omega^{*} \operatorname{Im}(H)}{1+M^{*} \operatorname{Re}(H)}, \\
K & =\frac{1}{1+M^{*} \operatorname{Re}(H)}, \quad A=\frac{\varepsilon^{*} \omega^{* 2}}{1+M^{*} \operatorname{Re}(H)}
\end{aligned}
$$

where $\delta^{*}$ is the maximum dimensionless amplitude. 
The cantilever shaft with the disk at the free end is rotating in a viscous medium. The schematic diagram for the rotor system is shown in Figure 22(b). The effects of cracks on the evolution of the bending frequencies as the speed of rotation (of cantilever rotor, see Figure 24 (a)) changes are evaluated using Campbell diagrams (Figures 24(b) and (c)).

\section{REFERENCES}

Behera, R.K., Parhi, D.R., and Sahu, S.K., 2004, "Vibration analysis of simply supported shaft with multi cracks," in Proceedings of the $3^{\text {rd }}$ International Conference on Vibration Engineering and Technology of Machinery (VETOMAC) and the $4^{\text {th }}$ Asia-Pacific Conference on System Integrity and Maintenance (ACSIM), December 6-9, pp.79-83.

Brennen, C., 1976, “On the flow in an annulus surrounding a whirling cylinder,” Journal of Fluid Mechanics 75 (1), 173-191.

Collins, K.L., Plaut, R.H., and Wauer, J., 1991, "Detection of cracks in rotating Timoshenko shaft using axial impulses," ASME Journal of Vibration and Acoustics 113, 74-78.

Fritz, R.J., 1970, “The effects of an annular fluid on the vibrations of a long rotor, part 1 - Theory," Journal of Basic Engineering 92 (4), 923-930.

Iida, S., 1958, Transactions of the Japan Society of Mechanical Engineers (in Japanese) 24 (141), 278-283.

Kadyrov, S.G., Wauer, J., and Sorokin, S.V., 2001, "A potential technique in the theory of interaction between a structure and a viscous, compressible fluid," Archive of Applied Mechanics 71, 405-417.

Kito, F., 1956, Transactions of the Japan Society of Mechanical Engineers (in Japanese) 22 (121), 663.

Ostachowicz, W.M. and Krawczuk, M., 1996, "Coupled torsional and bending vibrations of a rotor with an open crack," Archive of Applied Mechanics 62, 191-201.

Papadopoulos, C.A. and Dimarogonas, A.D., 1987, "Coupled bending and torsional vibration of a cracked Timoshenko shaft," Ingenieur Archiv 57, 257-266.

Papadopoulos, C.A. and Dimarogonas, A.D., 1987, "Coupled longitudinal and bending vibrations of a rotating shaft with an open crack," Journal of Sound and Vibration 117, 81-93.

Shimogo, T. and Kazao, Y., 1982, "Critical speed of rotor in a liquid," Bulletin of the Japan Society of Mechanical Engineers, 25 (200), 277-283.

Walston, W.H., Ames, W.F., and Clark, L.G., 1964, "Dynamic stability of rotating shafts in viscous fluids," ASME Journal of Applied Mechanics, 292-299.

Wauer, J., 1990, “On the dynamics of cracked rotors: A literature survey,” Applied Mechanics Review 43 (1), $13-17$.

Wauer, J., 1990, "Modeling and formulation of equations of motion for cracked rotating shafts," International Journal of Solids and Structures 26, 901-914.

Wauer, J. and Mehl, V., 1994, "Flow instability between coaxial rotating cylinders with a flexible support," in Series on Stability, Vibration and Control of Structures, Vol.1: Wave Motion, Intelligent Structures and Nonlinear Mechanics, A. Guran and D. J. Inman, eds., World Scientific, Hackensack, NJ, pp. 280-291. 